\title{
Dinámica Demográfica de Chile y América Latina: Una Vi- sión a Vuelo de Pájaro
}

MIGUEL VILLA

DANIELA GONZÁLEZ*

El propósito de este artículo es examinar la dinámica demográfica chilena ælos cambios en materia de crecimiento natural de la población, mortalidad, fecundidad y estructura por edadesæ en el contexto latinoamericano; el período de referencia abarca la segunda mitad del siglo XX y el primer quinquenio del XXI. Una tarea de esta naturaleza exige utilizar un amplio caudal de datos ${ }^{1}$; aunque ello es condición necesaria para dimensionar los fenómenos que se abordan, el precio es un texto duro y un tanto ríspido, en el que la gran cantidad de cifras y gráficos deja poco espacio al análisis cualitativo. Numerosas razones justifican el subtítulo del artículo, ya que se trata de una presentación sucinta de un panorama regional de gran heterogeneidad; la mirada desde la altura equivale a la que se tiene de un mapa a pequeña escala: impide percibir detalles, muchas veces importantes, para comprender los procesos sociales que subyacen al cambio demográfico, aunque puede prestarse como un medio de orientación general. La noción restringida de dinámica demográfica omite dos capítulos fundamentales: la movilidad (interna e internacional) y la distribución espacial de la población.

\section{El proceso de transición demográfica}

1.1. Atenuación del crecimiento. Cuando comenzó la segunda mitad del siglo XX, la población de Chile era de poco más de seis millones de personas; en 1985 excedió los 12 millones. La duplicación del número de efectivos, en apenas 35 años,

* Funcionarios del Centro Latinoamericano y Caribeño de Demograf́a (CELADE) æDivisión de Población de la Comisión Económica para América Latina y el Caribe (CEPAL). Las opiniones expresadas en este texto, que no ha sido sometido a revisión editorial, son de exclusiva responsabilidad de los autores y pueden no coincidir con las de la Organización.

1 El grueso de la información utilizada procede del banco de datos del CELADE (www.eclac.cl); las demás fuentes consultadas se indican en el texto y los gráficos. 
fue un hecho sin precedentes, que recibió el mote de "explosión demográfica", pero este ímpetu expansivo fue de corta duración, ya que no se mantuvo constante a lo largo del período. A raíz de la persistencia de niveles relativamente elevados de natalidad æque incluso tuvo un leve ascensoæ y de la rápida declinación de la mortalidad, la tasa de crecimiento demográfico vegetativo alcanzó su máximo histórico entre 1955 y 1965 (2,6\% por año). Sin embargo, en los años siguientes, cobró fuerza una tendencia contraria, pues la súbita baja de la fecundidad impulsó la caída de la tasa de crecimiento, que se redujo al 1,7\% en los años setenta y ochenta. La consolidación de esta segunda tendencia dio lugar a que el ritmo de incremento demográfico siguiera aminorando, para llegar al 1.1\% en el primer quinquenio del siglo XXI; en 2004 se estima que el país cuenta con una población de casi 16 millones de personas.

Si bien la evolución demográfica de Chile no constituye un caso excepcional en América Latina, sus cambios se han producido de acuerdo a tiempos y velocidades distintos a los advertidos en otros países (gráfico 1). Así, la mayor celeridad del crecimiento medio de la región ædesde 1950 en adelanteæ se tradujo en una reducción del peso relativo de la población chilena dentro del concierto regional: del $3,8 \%$ en 1950 bajó al $3.1 \%$ en 1985 y al $2.9 \%$ en 2004 . Otra indicación de la pérdida de tamaño relativo de la población chilena se obtiene de la comparación de su magnitud con la que presentan las dos subregiones más cercanas: la proporción de habitantes del país dentro del cono sur de América disminuyó del 7,5\% en 1950 al 6,5\% en 2004; dentro del ámbito andino, la caída fue aun más notoria, ya que pasó del $16,2 \%$ en 1950 al $11.7 \%$ en $2004 .^{2}$

\section{Gráfico 1}

Chile y américa latina: Población total y tasa de crecimiento, 1950-2000

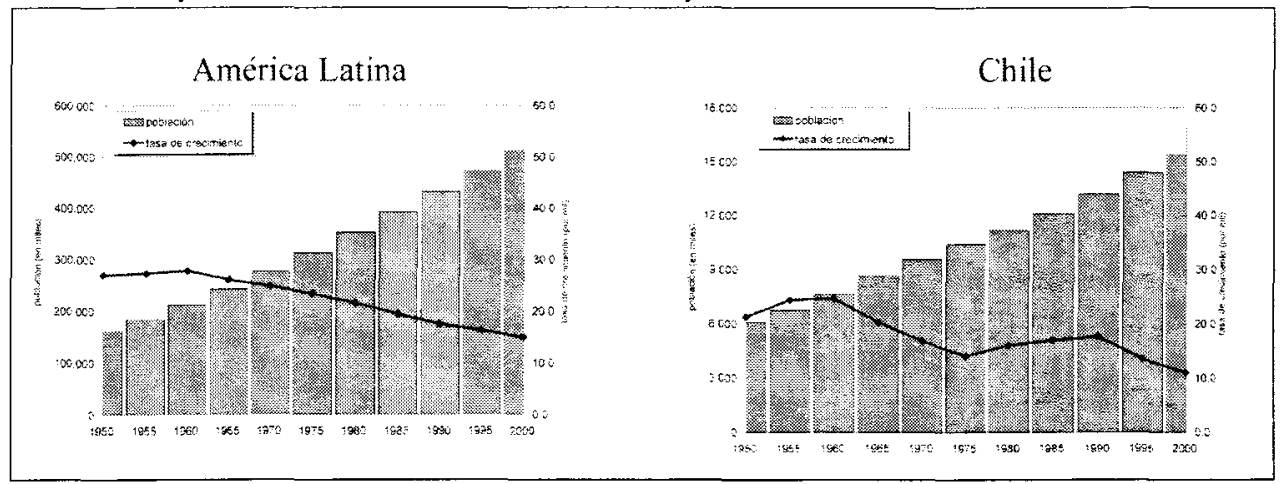

Fuente: Banco de datos del CELADE.

2 Para el cálculo de los respectivos porcentajes la población de Chile se incluye dentro del total de la que habita en el cono sur (Argentina, Brasil, Paraguay y Uruguay) y en el ámbito andino (Bolivia, Colombia, Ecuador y Venezuela). 
1.2. Transición demográfica, un esquema ordenador. Con el fin de examinar el dinamismo aparentemente menor de la población chilena en el contexto latinoamericano, es útil hacer uso del esquema analítico de la transición demográfica, que se concibió, inicialmente, para interpretar la transformación sociodemográfica de los países europeos durante su proceso de industrialización, entre mediados del siglo XVIII y del XX. En América Latina, así como en Asia, se ha podido advertir un proceso parecido, en sus formas, al registrado en los países europeos, pero estas experiencias de transición difieren considerablemente, y no sólo en sus expresiones temporales y espaciales, sino, principalmente, en lo que se refiere a sus sustratos sociales, económicos y culturales.

Despojado de un afán explicativo específico, dicho esquema comprende varias instancias que se prestan para describir las características básicas del cambio demográfico de los países en los últimos decenios. La primera instancia es la previa a la transición, caracterizada por tasas de natalidad y mortalidad elevadas, y con intensidades similares, que dejan una pequeña brecha para el incremento natural de la población. Durante una segunda etapa, propiamente de transición, la mortalidad inicia una caída secular, pero la natalidad, alimentada por ideales reproductivos asociados a familias de gran tamaño, sigue elevada; la combinación entre ambas tendencias origina un aumento de las tasas de crecimiento de la población. La tercera fase, de transición avanzada, se hace presente cuando declina la natalidad; por lo mismo, las tasas de crecimiento de la población se hacen cada vez más reducidas. En algunos casos extremos, las tasas de natalidad caen sistemáticamente por debajo de las de mortalidad, generando una situación de postransición, que se expresa en tasas negativas de incremento natural de la población. ${ }^{4}$

1.3. La transición demográfica en la región. La instancia de pretransición, de una muy elevada mortalidad y una alta fecundidad, se mantuvo vigente en los países de América Latina hasta mediados o fines del siglo XIX. Fue sólo durante el siglo $\mathrm{XX}$ que la población de la región dio señales de su ingreso al proceso de transición, con lo que se gestó el progresivo desmoronamiento del viejo orden demográfico.

3 La bibliografia que aborda la transición demográfica europea y de los países actualmente desarrollados es abundante (Coale y Watkins, 1986). También son numerosos los estudios acerca de la transición demográfica en las regiones en desarrollo (uno de tipo comparativo es el de Tabah, 1989). Cabe destacar que la IV Conferencia Latinoamericana de Población se dedicó exclusivamente al análisis de la transición en América Latina (ABEP/CELADE/IUSSP/PROLAP/SOMEDE, 1994).

4 Algunos investigadores estiman que la fuerte contracción de los ritmos reproductivos en algunos países europeos configura una "segunda transición demográfica", vinculada con las grandes transformaciones socioculturales que inciden en comportamientos centrados en el individuo y en nuevos patrones de nupcialidad y organización familiar (van de Kaa, 2001 y 1997). Una exploración de este proceso de postransición en América Latina se presenta en CELADE (2002) 
Un punto culminante de este proceso se advirtió en el decenio de 1960: el descenso de la mortalidad, iniciado en ańos anteriores, y la persistencia de los altos ritmos reproductivos, hicieron posible una inusitada aceleración del crecimiento natural, reflejado en una tasa del 2,8\%. Como contrapartida, a contar de la segunda mitad del decenio de 1960, la población latinoamericana comenzó a experimentar una paulatina reducción de su fecundidad que, combinada con una disminución todavía intensa de la mortalidad, dio por resultado que la tasa de crecimiento se fuera aminorando gradualmente en los años siguientes, hasta ubicarse por debajo del $2 \%$ en la segunda mitad del decenio de 1980.

Después de unos 35 años, América Latina, como un todo, dejaba atrás la etapa de expansión vertiginosa de su población; una muestra elocuente de ello es que la tasa de crecimiento disminuyó al 1,5\% en el primer quinquenio del siglo XXI. Es decir, y no obstante las radicales diferencias entre las condiciones socioeconómicas de América Latina y las que prevalecían en Europa durante su transición demográfica, la celeridad de este proceso ha sido mucho mayor en la región, la que en medio siglo obtuvo un efecto parecido al que Europa logró en dos siglos. ${ }^{5}$ Buena parte de la diferencia, que hace sospechar de una creciente autonomía relativa de la esfera demográfica, radica en que los medios instrumentales, especialmente en el campo médico sanitario, de aquella transformación en América Latina tuvieron un desarrollo exógeno; además, algunos de esos medios, como los procedimientos modernos de anticoncepción, se concibieron después de que la población europea concluyó las etapas centrales de la transición.

1.4. Diversos cursos de transición. Debe tenerse presente que el panorama de transición descrito, válido para el conjunto de América Latina, no revela la gran heterogeneidad existente dentro de la región, que se expresa tanto en las posiciones de los países respecto de los indicadores básicos en un momento dado como en su velocidad de cambio. Esta heterogeneidad puede examinarse mediante un juego de diagramas acotados por los valores de las tasas brutas de natalidad y mortalidad, que determinan el crecimiento natural de la población.

Como se nota en el primer diagrama, el estado de la transición demográfica en el quinquenio 1950-55 era muy incipiente y se distinguía por la gran dispersión de los valores de las tasas nacionales de natalidad y mortalidad en torno a unos promedios regionales de por sí elevados (gráfico 2). En el extremo inferior se ubican Uruguay, Argentina y Cuba, países inmersos de lleno en el proceso de transición, que habían logrado importantes progresos en la reducción de la mortalidad y tenían los meno-

5 Otro aspecto de diferenciación estriba en que Europa nunca llegó a tener tasas de natalidad tan elevadas como las que se registraron en América Latina. 
res niveles de natalidad de la región; sus tasas de crecimiento natural, inferiores al $2 \%$, y casi del $1 \%$ en Uruguay, equivalían a las que alcanzó la población latinoamericana recién a fines de los años ochenta. Otros cinco países, que también registraban avances, notorios en Paraguay y menores en Chile, en la lucha contra la mortalidad, integraban un segundo grupo, con un amplio rango de tasas de natalidad, que oscilaban entre valores inferiores a la media en Chile y Panamá, y otros que la superaban con creces en Venezuela y Costa Rica; a raíz de estas variaciones, las tasas de crecimiento oscilaban entre el 2,3\% en Chile y más del 3\% en Venezuela, Paraguay y Costa Rica. Un tercer conjunto estaba constituido por tres países que concentraban el 59\% de la población regional, por lo que no sorprende que sus tasas vitales fuesen similares a las medias de la región; con todo, sus niveles de natalidad, algo por arriba del promedio, salvo en el caso de Brasil, se traducían en un ritmo de crecimiento natural ligeramente más alto que el del conjunto regional. El grupo más numeroso de países, a la derecha de las medias de América Latina, presentaba una situación de alta mortalidad y natalidad, con tasas de crecimiento natural particularmente elevadas en las naciones centroamericanas y algo menores en las andinas. Por último, Bolivia y Haití parecían compartir una condición de pretransición; sus tasas de crecimiento inferiores al promedio revelaban los efectos de una muy alta mortalidad, que tronchaba el impacto potencial de la natalidad.

El diagrama correspondiente al quinquenio 1975-1980, se caracteriza por un generalizado desplazamiento de las tasas vitales hacia valores claramente inferiores a los observados en los años cincuenta, cambio que se aprecia en la fuerte disminución de la mortalidad y el descenso algo menos marcado de la natalidad; es decir, a comienzos del último tercio del siglo XX, la mayoría de los países latinoamericanos había entrado a una etapa de transición demográfica plena o avanzada (gráfico 3). El grupo que exhibía las tasas más bajas de natalidad seguía integrado por Uruguay, Argentina y Cuba, a los que se había agregado Chile; a raíz de las diferencias entre los ritmos reproductivos y de la diferente incidencia de la mortalidad, las tasas de crecimiento natural de Cuba y Uruguay eran inferiores a la unidad, pero las de Chile y Argentina rondaban el 1,7\%. Un segundo conjunto estaba conformado por el mayor número de países, en los que residía bastante más de los dos tercios de la población latinoamericana, con niveles de mortalidad inferiores al promedio æsalvo en el caso de Brasilæ y de natalidad similares a la media regional; sus tasas de crecimiento vegetativo oscilaban entre el 2,4\% en Brasil y el 2,9\% en México. El tercer grupo se distinguía por niveles de mortalidad y natalidad claramente superiores a los de América Latina, y sus tasas de crecimiento iban desde el $2,7 \%$ en Perú y el $2,8 \%$ en Ecuador hasta el 3,4\% en Honduras y Nicaragua. Si bien Bolivia y Haití seguían ubicados en una posición alejada del resto de los países, registraban descensos claros de la mortalidad y una leve insinuación de baja de la natalidad, por lo que 
habían ingresado a una etapa de transición incipiente, con tasas de crecimiento del $2,5 \%$, semejantes al promedio regional.

La información acerca del estado de la transición demográfica en el quinquenio 2000-05 deja en evidencia la consolidación del proceso en América Latina; las tasas de natalidad y mortalidad tienen límites superiores claramente más reducidos que en los años ochenta y, salvo por unas pocas situaciones nacionales en posiciones de rezago relativo, la vieja imagen de dispersión se ha convertido en una de aparente convergencia (gráfico 4). En relación con el período precedente, los cambios más importantes corresponden a la natalidad. Cuba alcanza valores que parecen propios de un estado de postransición, con un muy bajo nivel de natalidad y una tasa de mortalidad en ascenso, rasgo inherente a una población envejecida, que origina un ritmo de crecimiento natural de sólo $0,4 \%$; Argentina y, sobre todo, Uruguay revelan los efectos de una transición prolongada, y en Chile se observa una marcada declinación de la tasa de crecimiento de la población. En el otro extremo, Haití, Bolivia y Guatemala, comparten niveles de natalidad y mortalidad mayores que el promedio, pero difieren en su ritmo de crecimiento: la tasa de Guatemala (3\%), a raíz de la persistencia de una elevada fecundidad, duplica la media regional; las menores tasas de Bolivia y, en especial, de Haití, ambas similares a la media regional, acusan los efectos de una elevada mortalidad.

\section{Gráfico 2}

América Latina: grupo de países según su situación de transición demográfica, 1950-1955

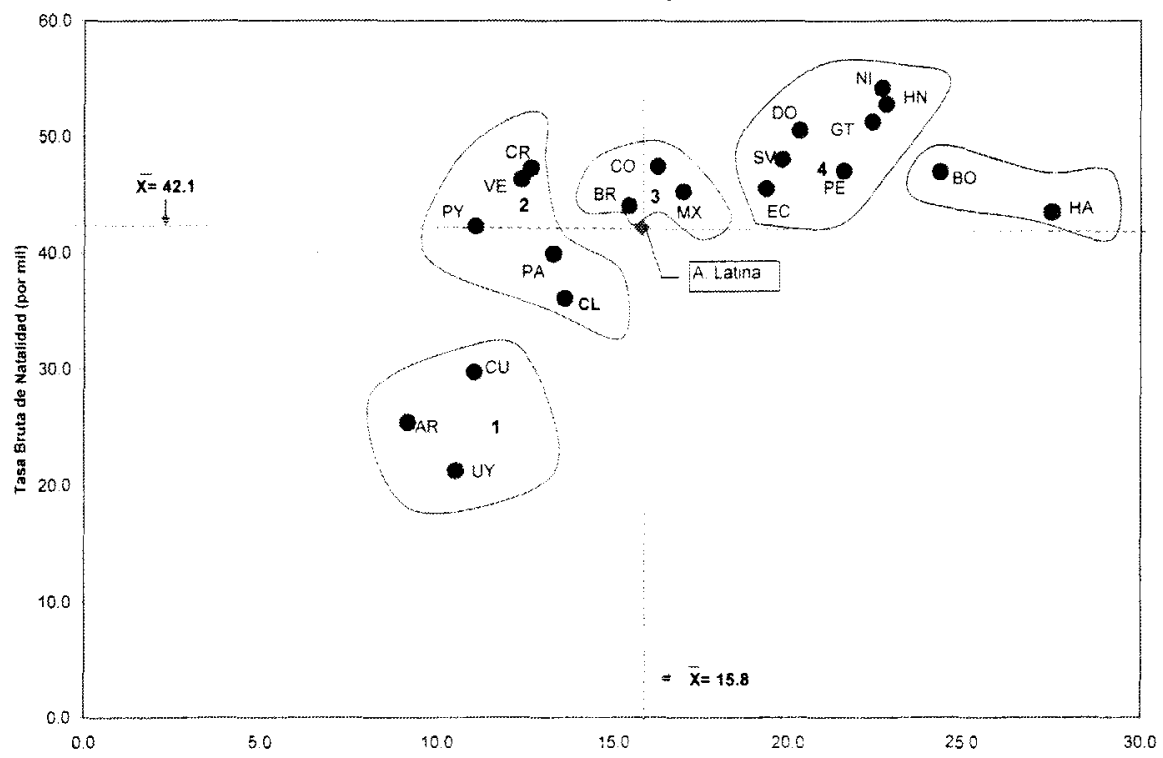

Fuente: Banco de datos del CELADE. 


\section{Gráfico 3}

América Latina: grupo de países según su situación de transición demográfica, $1975-1980$

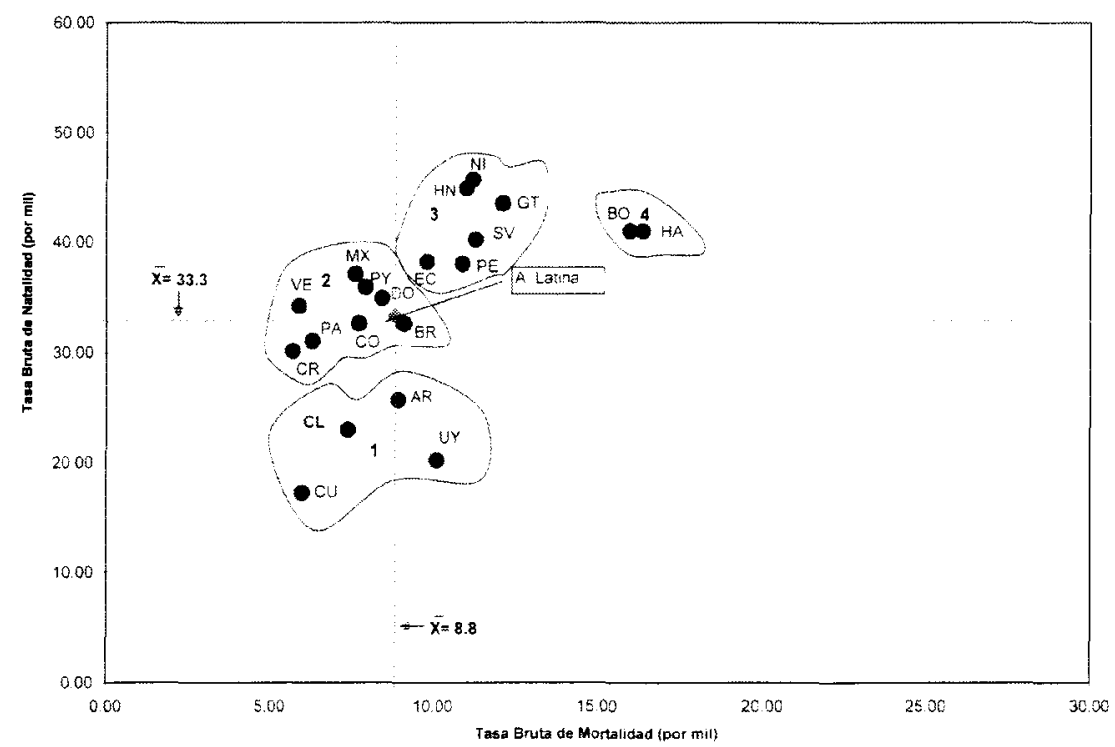

Fuente: Banco de datos del CELADE.

\section{Gráfico 4}

América Latina: grupo de países según su situación de transición demográfica, 2000-2005

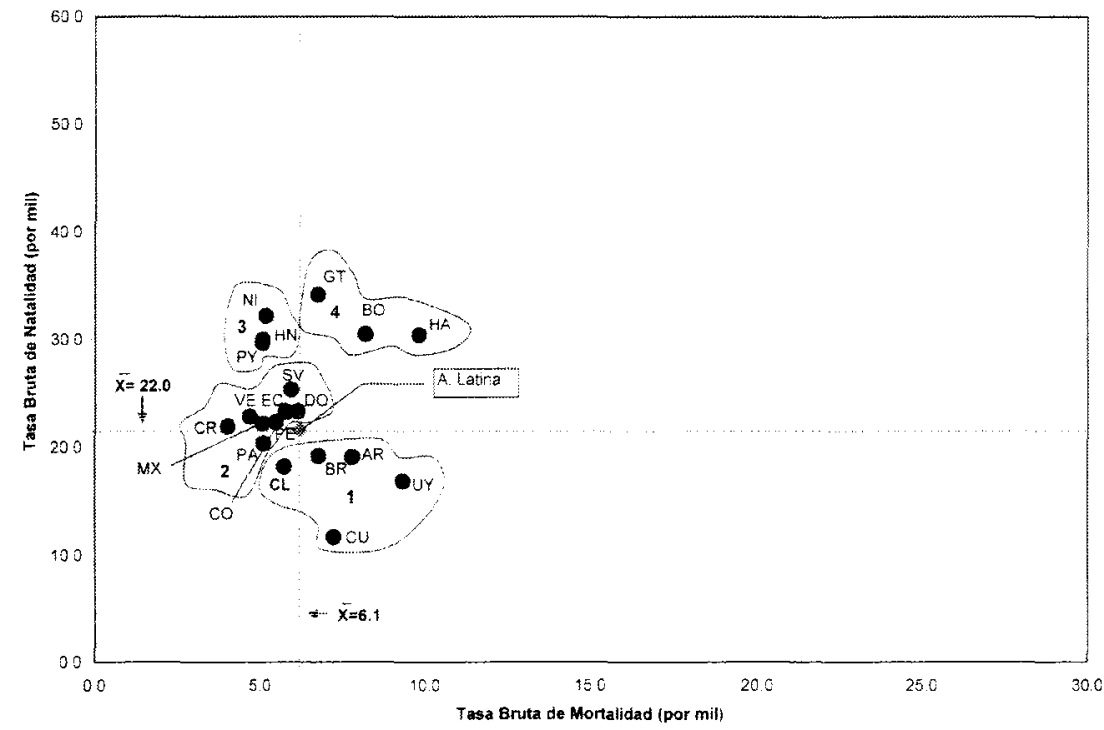

Fuente: Banco de datos del CELADE. 
1.5. Trayectorias específicas: divergencia y concordancia. Una forma diferente de identificar la posición de Chile en el contexto de los diversos cursos seguidos por el proceso de transición en América Latina, consiste en examinar las trayectorias nacionales a lo largo del tiempo. Con el propósito de brindar una visión de conjunto, y obviar detalles farragosos, sólo se consideran cinco países representativos de situaciones disímiles, y el promedio regional, que sirve de elemento de referencia (gráfico 5). Entre aquellos países, Argentina aparece como precursor de la transición, ya que los valores de sus tasas vitales a comienzos de los años cincuenta, coinciden con los que la región consiguió mucho más tarde: en los setenta, en el caso de la mortalidad, y recién en los noventa, en el de la fecundidad; con todo, las modificaciones ocurridas después del decenio de 1950 han sido pausados, rasgo que en parte responde al gradual envejecimiento de la estructura de edades. El modelo argentino de cambio demográfico, similar al uruguayo, estuvo profundamente influido por la inmigración europea, cuyos efectos se hicieron sentir desde fines del siglo XIX. En el caso de Cuba, se aprecia un considerable avance en la reducción de la mortalidad, que es particularmente ostensible en el primer intervalo (1950-1980); en los años siguientes, la abrupta caída de las tasas de natalidad, fruto de un drástico cambio en los patrones de comportamiento reproductivo, repercutió en una fuerte desaceleración del crecimiento de la población. Si bien las tasas vitales de Cuba arrancaron de valores moderados en los años cincuenta, su dinámica de cambio, especialmente en los últimos decenios, ha sido mucho más acentuada que la observada en Argentina.

\section{Gráfico 5}

Chile y otros países latinoamericanos: trayectoria de la transición demográfica,

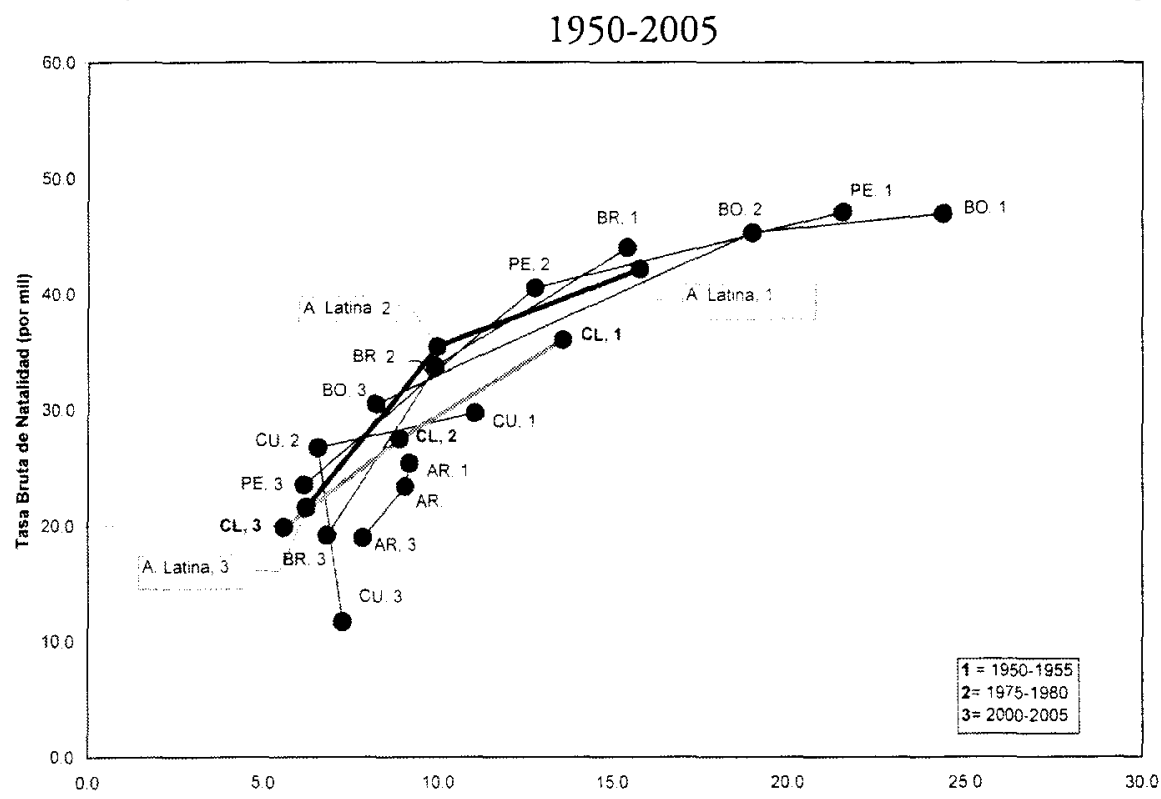

Fuente: Banco de datos del CELADE. 
Como cabe esperar de su considerable peso demográfico, la trayectoria de Brasil guarda un estrecho parecido a la del promedio de América Latina, sólo que se distingue de ésta por el acelerado descenso de sus niveles de natalidad durante el segundo período (1980-2005); menos significativos han sido los avances en materia de disminución de la mortalidad. Las cifras de Perú ilustran el paso de una situación de rezago relativo en los años cincuenta, cuando predominaban muy altas tasas vitales, a otra de transición moderada en el decenio de 1970, avance que encuentra su explicación en la baja de la mortalidad; la tasa bruta de natalidad, que se había mantenido casi sin variación en esa primera etapa, descendió con fuerza al final. El caso de Bolivia se distingue por un inicio más tardío de la transición, pues sus modificaciones principales ocurrieron después del quinquenio 1975-80, cuando las tasas de mortalidad se redujeron a niveles moderados, y las de natalidad comenzaron a decrecer.

La trayectoria de la transición demográfica chilena tiene rasgos específicos, que la sitúan en una posición intermedia respecto de los otros cinco países. Los valores de sus tasas vitales siempre estuvieron por debajo del promedio regional, aunque fueron superiores a los de Argentina y Cuba. El ritmo de cambio a lo largo de los cincuenta años fue más acentuado que el de Argentina, pero menos drástico que el de Cuba. Al final del período de observación se aprecia una mayor cercanía con los indicadores de Brasil, salvo que en este país aún existe una mayor mortalidad. Sin duda que el distingo mayor es el que puede establecerse con Bolivia; menos marcado es el contraste con Perú, especialmente en el último quinquenio.

\section{Evolución de la mortalidad y la fecundidad}

2.1. Prolongación de la vida. El valor de la tasa bruta de mortalidad de Uruguay, a fines del siglo XX, muy superior a la media de la región y sólo inferior a la cifra de Haití, pone una nota de alerta acerca de las restricciones que afectan a esta tasa, en tanto indicador de la mortalidad y de las condiciones de salud de un pás. Dicho valor responde al efecto de una larga y sostenida historia de baja fecundidad, en virtud de lo cual una alta proporción de la población uruguaya se ubica en los estratos de edades avanzadas, entre las cuales ocurre la mayoría de las muertes. Algo similar puede decirse de la tendencia ascendente de la tasa bruta de mortalidad de Cuba en los últimos años, puesto que se hace eco de una drástica reducción de la fecundidad, que incide en el veloz envejecimiento de la población. Por ende, para una comparación más rigurosa entre los países, es preferible utilizar la esperanza de vida al nacer (EVN), indicador libre del efecto de distorsión asociado a las diferencias entre las estructuras por edad, pero sensible a los niveles de la mortalidad en la infancia y la niñez.

La EVN del conjunto de la población de América Latina aumentó de 52 a 72 años entre comienzos del decenio de 1950 y el primer quinquenio del siglo XXI 
(gráfico 6). Esta notable ganancia de casi medio año de vida por cada año calendario, redujo de modo significativo la brecha de mortalidad que tradicionalmente ha separado la región en relación a las de mayor desarrollo. ${ }^{6}$ Este progreso en el control de la morbimortalidad se consiguió mediante el acceso a una tecnología adecuada para la prevención y el tratamiento de las enfermedades infecciosas y parasitarias, que causaban una elevada proporción de las defunciones, particularmente entre los niños de corta edad. Los programas de vacunación masiva y de erradicación de la malaria, permitieron evitar muchas muertes causadas por el paludismo, la difteria, el tétano y el tifus; asimismo, la disponibilidad de los antibióticos hizo posible la reducción de la mortalidad originada por la tuberculosis, la neumonía y la influenza. La institucionalización de los servicios de sanidad pública, esfera en la que Chile tuvo un papel precursor en América Latina, y las medidas dirigidas a prevenir y tratar las afecciones contagiosas, provocaron un considerable efecto positivo, incluso en las zonas donde se registraban deficientes condiciones de vida. Con todo, es forzoso reconocer que la mayoría de los programas de atención a la salud pública dependieron menos de la situación económica de los países que de la importación de tecnología generada en los países desarrollados (al decir de Sauvy, "una inyección de civilización").

\section{Gráfico 6}

Chile y otros países latinoamericanos: esperanza de vida al nacer, 1950-2005

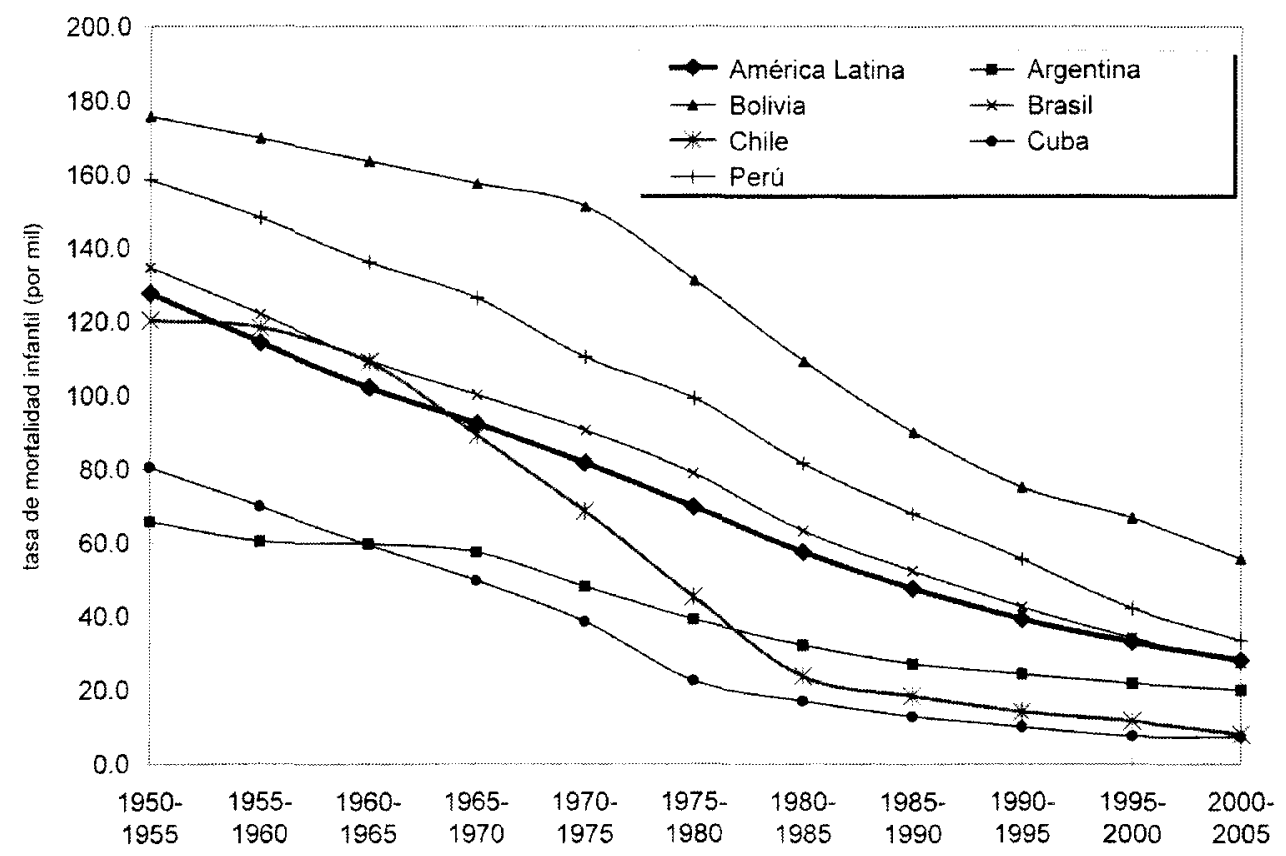

Fuente: Banco de datos del CELADE.

6 En 1950-55, la EVN media de América Latina era inferior en 14 años a la de Europa; en 2000-05, esa diferencia se acortó a sólo dos años (United Nations, 2003). 
No obstante el gran aumento de la EVN en la región, la heterogeneidad de situaciones entre los países redunda en grandes diferencias. Así, en 1950, los valores nacionales fluctuaban entre 66 en Uruguay y 38 en Haití; la cifra en Chile era de casi 55 años. En 1970, cuando el promedio latinoamericano ascendió a 61 años, el rango de variación iba desde los 69 años en Uruguay hasta los 47 en Bolivia; en Chile el indicador se aproximaba a 64 años. En el primer quinquenio del siglo XXI, el elevado promedio regional de 72 años está acompañado de un amplio campo de variación entre los países de mayor y menor mortalidad: Haití, en un extremo, con una EVN de 59 años (siete menos que la EVN de Uruguay en 1950); en el otro, Costa Rica, Chile y Cuba, con 77 a 78 años.

2.2. Mortalidad y transición epidemiológica. Desde los años setenta se advierte una gradual desaceleración del ritmo de incremento de la EVN, que guarda relación con un radical cambio de las principales causas de muerte y de la estructura por edad de las defunciones. En tal sentido, la declinación de la mortalidad trasunta una transición epidemiológica: al tiempo que se baten en retirada las causas de tipo infeccioso y parasitario, adquieren mayor ponderación las de índole crónica y degenerativa, como el cáncer, las afecciones cardiovasculares y los accidentes. ${ }^{7}$ Por ejemplo, en Chile, Costa Rica y Cuba, las enfermedades contagiosas representaban más de un tercio de las muertes en los años cincuenta, pero en los noventa su incidencia disminuyó a menos del 5\%; sin embargo, la mortalidad imputable a tales patologías sigue elevada en los países de baja EVN, como Guatemala, donde todavía en los años noventa, causaron más del $40 \%$ de las muertes (Chackiel, 2004). Además, el efecto de las enfermedades contagiosas aún es muy alto entre los grupos sociales en posición desmedrada: personas de ingresos bajos y menor grado de educación, residentes en áreas rurales y, en especial, las que pertenecen a los pueblos indígenas.

No puede desconocerse que el avance de la transición epidemiológica ha sufrido reveses, como lo revela la reaparición de enfermedades que se suponía controladas, tales como la malaria, el dengue, el cólera, la fiebre amarilla, la peste bubónica y el virus hanta, y cuya renovada intensidad pareciera deberse al debilitamiento de los sistemas nacionales de salubridad, así como al deterioro de los programas de prevención, las deficiencias de la educación para la salud, la contaminación de las fuentes de agua y el hacinamiento en algunas zonas urbanas (OPS, 2002). En definitiva, si bien el brinco inicial de la EVN estuvo ligado a la importación de tecnologías médicas y a los avances en materia de salubridad, los progresos futuros dependerán de una elevación de las condiciones materiales de vida de los

7 El creciente peso relativo de las enfermedades crónicas se vincula con el envejecimiento de la población. 
grupos más desposeídos de la región, lo que exigirá esfuerzos especiales en materia de empleo, distribución del ingreso, acceso a educación y servicios médicos de calidad, mejoras nutricionales, disponibilidad de agua potable y saneamiento ambiental. Además, será necesario desarrollar campañas de naturaleza preventiva para hacer frente a nuevas enfermedades transmisibles, como el VIH/SIDA, que ha cobrado una importante difusión en varios países de la región. ${ }^{8}$

2.3. Aumenta la supervivencia infantil. Gran parte del aumento de la EVN en las décadas pasadas se consiguió gracias al incremento de la supervivencia de los niños menores de cinco años, en especial de los infantes (gráfico 7). A comienzos del decenio de 1950, la tasa de mortalidad infantil (TMI) de la región indicaba que 128 de cada mil niños nacidos vivos, fallecían antes de enterar su primer cumpleaños. El hecho de que el indicador fuera mayor que 150 por mil en siete países y que sólo en cuatro se ubicara bajo 100 por mil, pone en evidencia la muy elevada frecuencia de las defunciones entre los niños latinoamericanos. Chile no era una excepción, su TMI de 120 por mil en el quinquenio 1950-55 coincidía casi exactamente con el promedio regional.

En la segunda mitad de los años setenta, y en parte como fruto de los esfuerzos en el campo de la atención de la salud materno infantil, la TMI de América Latina disminuyó al 70 por mil. Mayor fue el descenso en el caso de Chile, ya que su tasa del 45 por mil era un $65 \%$ inferior a la registrada en los años cincuenta. La "maduración" de las inversiones en salud, el establecimiento de programas de nutrición complementaria, los adelantos en materia de educación de las madres y el descenso de la fecundidad, con la consecuente reducción de los riesgos asociados a los altos ritmos reproductivos del pasado, contribuyeron a una todavía más drástica caída de la TMI en los decenios siguientes. En el primer quinquenio del siglo XXI, el indicador regional es de 28 por mil, pero aún hay grandes diferencias entre los países: en ocho se registran valores por encima de 30 por mil (incluso en dos se advierten cifras superiores a 50 por mil); otros, en cambio, han logrado tasas inferiores a la mitad del valor regional. Entre estos últimos se destacan Cuba, Chile y Costa Rica, con tasas que van del 7 al 11 por mil y que se asemejan a las del conjunto de los países europeos (United Nations, 2003).

8 Se estima que en el año 2000 el $0.6 \%$ de la población latinoamericana de 15 a 49 años vivía con esta enfermedad (Chackiel, 2004). 


\section{Gráfico 7}

Chile y otros países latinoamericanos: tasa de mortalidad infantil, 1950-2005

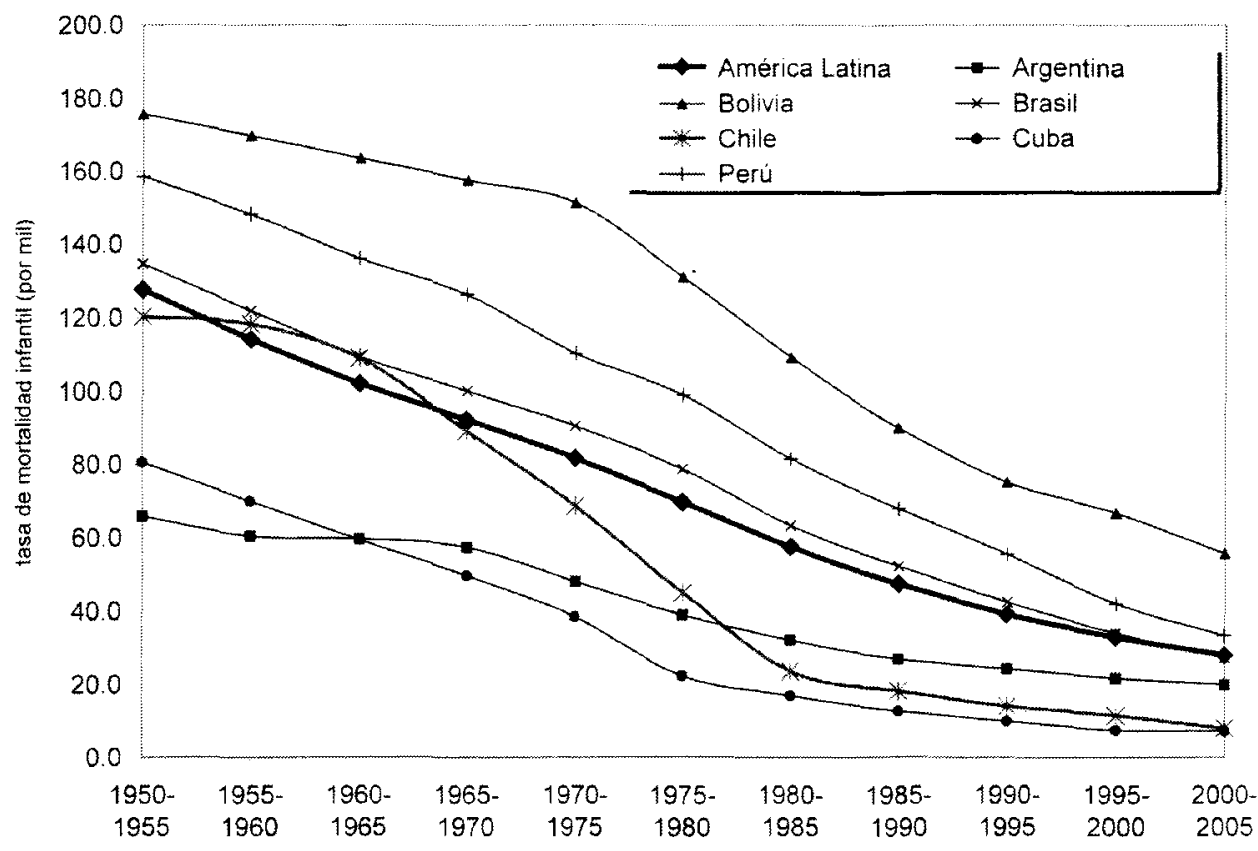

Fuente: Banco de datos del CELADE.

Una de las evidencias empíricas de los riesgos del rezago de la transición demográfica, es la persistencia de la alta mortalidad infantil, que atenta contra el más elemental de los derechos. Sin embargo, es necesario destacar que dicho rezago no sólo se manifiesta entre los países, ya que también se manifiesta con fuerza dentro de los mismos. Un rasgo característico de América Latina, que la distingue de las demás regiones en desarrollo, es su profunda y sostenida desigualdad social (CEPAL, 2004b), patente en las acusadas inequidades sociodemográficas. En lo que se refiere a mortalidad infantil, las diferencias entre los diversos estratos sociales cobran múltiples expresiones.

Si bien la TMI es claramente más elevada en los países donde la transición demográfica se encuentra en etapas incipientes o moderadas, los valores medios nacionales no permiten advertir la situación de los grupos sociales en condiciones de desventaja, principalmente los estratos bajos urbanos, los grupos campesinos y las comunidades indígenas. Así, por ejemplo, en la mayoría de los países la mortalidad de los hijos de madres que carecen de instrucción, duplica la que registran los hijos de madres con educación secundaria o superior (gráfico 8). Estas desigualdades no 
sólo se aprecian en los países que se encuentran en etapas iniciales de la transición demográfica, como lo ilustran los datos de Bolivia, sino también en aquellos otros que registran avances mayores en el proceso, como es el caso de Brasil. En Chile, en cambio, el descenso de la TMI se ha generalizado entre los distintos grupos sociales, condición que, con diferentes intensidades, también parece presente en El Salvador y Honduras, lo que revela que un riesgo sociodemográfico puede reducirse con alguna autonomía respecto de los contextos socioeconómicos, del grado de desarrollo y de la equidad social. ${ }^{9}$

\section{Gráfico 8}

Chile y otros países latinoamericanos: tasa de mortalidad infantil según nivel de instrucción de la madre, 1950-2005

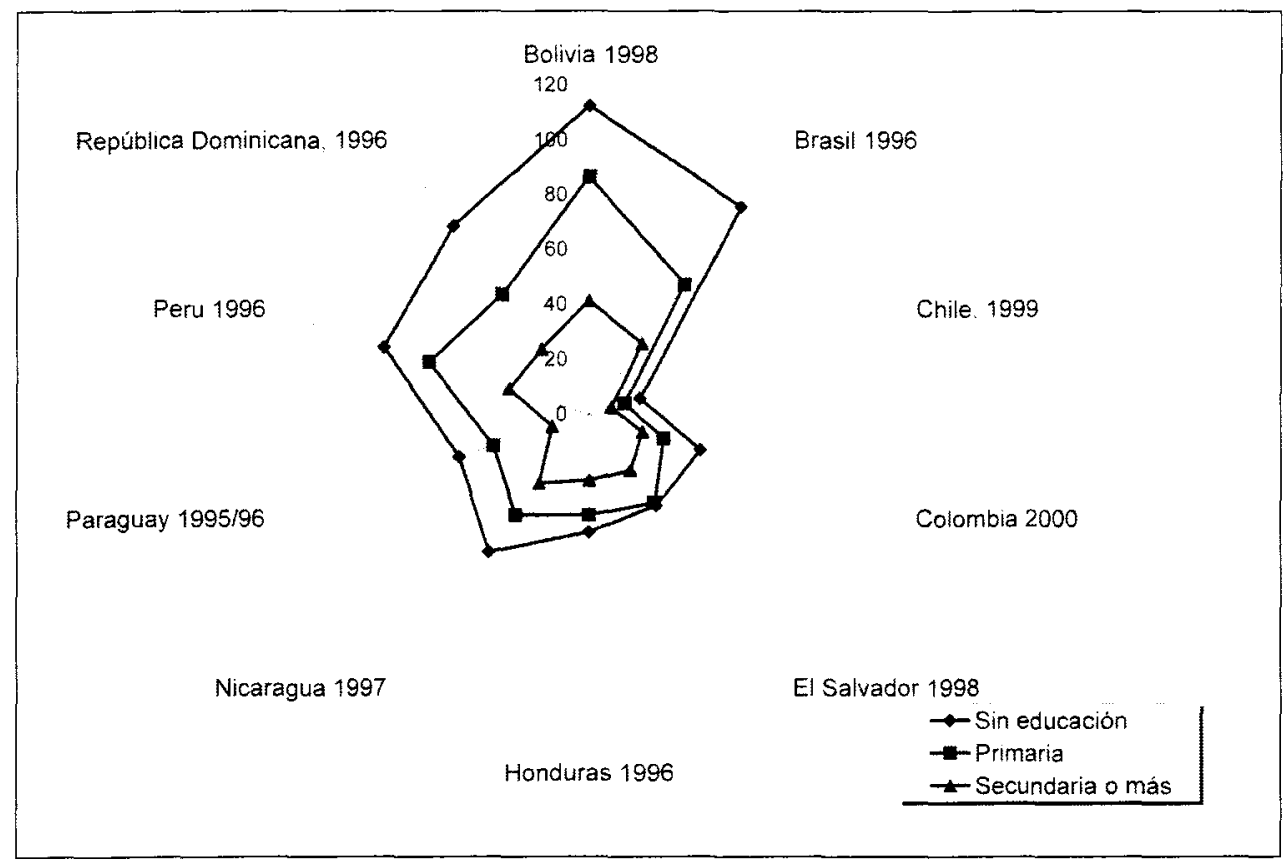

Fuente: CEPAL/CELADE (2002)

9 Según Chackiel (2004), la mortalidad infantil ha descendido en todos los países "ndependientemente de los indicadores sociales y económicos, y sin que se aprecien retrocesos o un freno en la velocidad de la caída de las tasas en los momentos de crisis económica como las ocurridas durante la década de 2980 " (p. 41). 
2.4. Transformación de los comportamientos reproductivos. ${ }^{10}$ La más profunda transformación sociodemográfica de América Latina en el último siglo, ha sido el descenso de la fecundidad, que se inició en la segunda mitad del decenio de 1960 y que responde a una constelación de factores determinantes. Durante el período de posguerra, y hasta comienzos del decenio de 1970, la economía regional, dinamizada por el proceso de sustitución de importaciones, con el protagonismo de la industria y los servicios, registró una sostenida expansión, que se tradujo en un incremento relativamente sostenido del producto per capita. Al amparo de esta expansión, se gestaron profundas modificaciones sociales, como la ampliación de los estratos medios y asalariados, la extensión de la escolaridad y una veloz urbanización. Los cambios de las estructuras económicas y sociales trajeron aparejada una serie de mudanzas culturales, que contribuyeron a la adopción de pautas de comportamiento reproductivo compatibles con un ideal de familia de tamaño más reducido, aspiración facilitada por la disponibilidad de procedimientos anticonceptivos. ${ }^{11}$

La decisión de optar por una descendencia menos numerosa se vio reforzada por un cambio en la condición de los hijos dentro de los nuevos contextos sociales, económicos y culturales. La contribución de los hijos al sostén familiar, que tenía especial importancia en el ámbito campesino y las formas tradicionales de organización societaria, fue disminuyendo gradualmente, al tiempo que se elevaban sus costos de crianza, tanto en virtud de la extensión de la escolaridad, requisito que se fue haciendo indispensable para el desempeño social, como a ráz del progresivo cambio en favor de un modelo de responsabilidades compartidas del hombre y la mujer, tanto dentro como fuera del hogar. En tal sentido, la mayor inserción femenina en el mundo del trabajo parece haber generado, por lo menos entre los estratos medios y altos, una incompatibilidad con las funciones estrictamente domésticas. Otro aspecto que merece ser considerado es el efecto de la disminución de la mortalidad infantil, pues es probable que la mayor probabilidad de supervivencia de los hijos fuese un motivo para obviar concepciones adicionales.

No puede ignorarse que la puesta en práctica de la anticoncepción fue un agente que facilitó la velocidad del descenso de los ritmos reproductivos en la región. A comienzos de los años sesenta, el acceso a los procedimientos anticonceptivos modernos se limitaba sólo a sectores reducidos de la población, principalmente los estratos medios y medios altos de las zonas urbanas. Con todo, diversas instituciones privadas, algunas imbuidas

10 En esta sección se usará la tasa global de fecundidad (TGF), un indicador del nivel de la fecundidad que es apropiado para efectuar comparaciones entre las poblaciones de distintos países. En cambio, la tasa bruta de natalidad æque mide la frecuencia relativa de los nacimientos con respecto a la población total, y no sólo de aquella que está expuesta al riesgo de procrear (la población femenina en edades fértiles)æ se ve afectada por el efecto de las diferencias en las estructuras por sexo y edad.

11 La transición de la fecundidad y los cambios en los comportamientos reproductivos han sido objeto de una amplia investigación. Entre los estudios precursores sobre la materia, cabe mencionar los de Urzúa (1979), González y otros (1982) y CEPAL (1984). Un tratamiento reciente realiza Guzmán (1997). 
por criterios neomalthusianas, desarrollaron intensas campañas de promoción. ${ }^{12} \mathrm{Al}$ mismo tiempo, diversos estudios médicos brindaban evidencias acerca del serio problema de salud inherente al aborto inducido, que se practicaba en condiciones de absoluta desprotección y gran riesgo. Estos antecedentes llevaron a que las autoridades de los organismos nacionales de salud de algunos países, entre los cuales se encontraba Chile, resolvieran incorporar programas de planificación familiar como componentes de la atención a la salud materno infantil. ${ }^{13}$ Más tarde, incluso antes de la celebración de la Conferencia Internacional sobre la Población y el Desarrollo (El Cairo, 1994), se reconoció el derecho de los individuos y las parejas a decidir respecto del número de hijos que deseen procrear y del momento apropiado para engendrarlos. Después de realizada la citada Conferencia, la mayoría de los países adoptó un modelo de salud reproductiva y sexual compatible con los derechos reproductivos (CEPAL/CELADE, 2004).

2.5. Una declinación abrupta. La fecundidad se mantuvo elevada en la mayoría de los países de América Latina hasta mediados del decenio de 1960, cuando las mujeres tenían, en promedio seis hijos. Incluso hay evidencias de que, tanto debido a la rápida baja de la mortalidad, que permitió reducir la esterilidad por causas patológicas y las muertes entre mujeres en edad fértil, como por el inicio más precoz de las uniones maritales, la fecundidad aumentó en varios países, como Chile, entre 1940 y 1960. Sin embargo, durante la segunda mitad de los años sesenta se gestó un abrupto cambio de la tendencia; en apenas 30 años, la tasa global de fecundidad (TGF) regional disminuyó a la mitad. Con un promedio de 2,6 hijos por mujer en el quinquenio 2000-2005. América Latina alcanzó una cifra similar a la que se registró medio siglo antes en los países europeos.

Dentro de la región se advierten grandes diferencias, tanto respecto del momento en que comenzó la reducción de la fecundidad como de la intensidad de este proceso (gráfico 9). Brasil y Perú, en razón de su peso demográfico relativo, han marcado la tónica de la transición reproductiva de América Latina. Brasil se destaca por la celeridad del descenso a contar de 1980, lo que condujo a un promedio de 2,3 hijos por mujer a principios del siglo XXI; si bien en Perú se aprecia un ritmo sostenido de declinación, la TGF se ha mantenido por encima de la media regional. La transición comenzó algo más tarde, y avanzó de manera más moderada, en Bolivia, por lo que el promedio de hijos por mujer sigue siendo elevado (casi 4 en el primer quinquenio del siglo XXI).

12 Estas campañas, apoyadas por novedosas estrategias de comunicación, parecen haber constituido un factor coadyuvante de los cambios en el comportamiento reproductivo.

13 El Servicio Nacional de Salud de Chile instituyó un programa de planificación familiar en 1963; al cabo de trece años de operación la cobertura efectiva alcanzaba a un quinto de la población total de mujeres en edad fértil (Soto, 1978). Sin embargo, la política de población adoptada por la Oficina de Planificación Nacional en 1978, inspirada en una lectura geopolítica de los cambios sociodemográficos, sostuvo que la reproducción no podía ser resorte del Estado, pero que la "alarmante crisis" ocasionada por la sustancial reducción de los nacimientos justificaba campañas destinadas a "dignificar y estimular la maternidad" (Population and Development Review, 1979, pp. 563-571); la respuesta oficial a una encuesta realizada en 1983 por las Naciones Unidas concuerda con esa posición (United Nations, 1985). 
Argentina es un caso atípico. Como fruto de su temprana transición demográfica, la TGF registrada en 1950 coincide con la recientemente alcanzada por la región como un todo en el decenio de 1990, pero los cambios netos a lo largo del período han sido escasos: un ligero aumento en el decenio 1970 seguido de una pausada disminución. El nivel de fecundidad de Chile æalgo menor que el promedio regional en $1950 x$ aumentó antes de descender de manera muy acelerada durante los años sesenta y setenta, para estabilizarse después, pero en los noventa volvió a disminuir; a raíz de esta evolución, la TGF de Chile llegó a 2 en el quinquenio 2000$2005^{14}$. Aún más notable que el de Chile es el caso de Cuba, pues su fecundidad también aumentó con fuerza a comienzos del decenio de 1960 y luego se redujo de manera drástica hasta llegar un nivel inferior al de reemplazo en 1980; en los años noventa el promedio de hijos por mujer era de apenas $1,6 .{ }^{15}$

\section{Gráfico 9}

Chile y otros países latinoamericanos: tasa global de fecundidad, 1950-2005

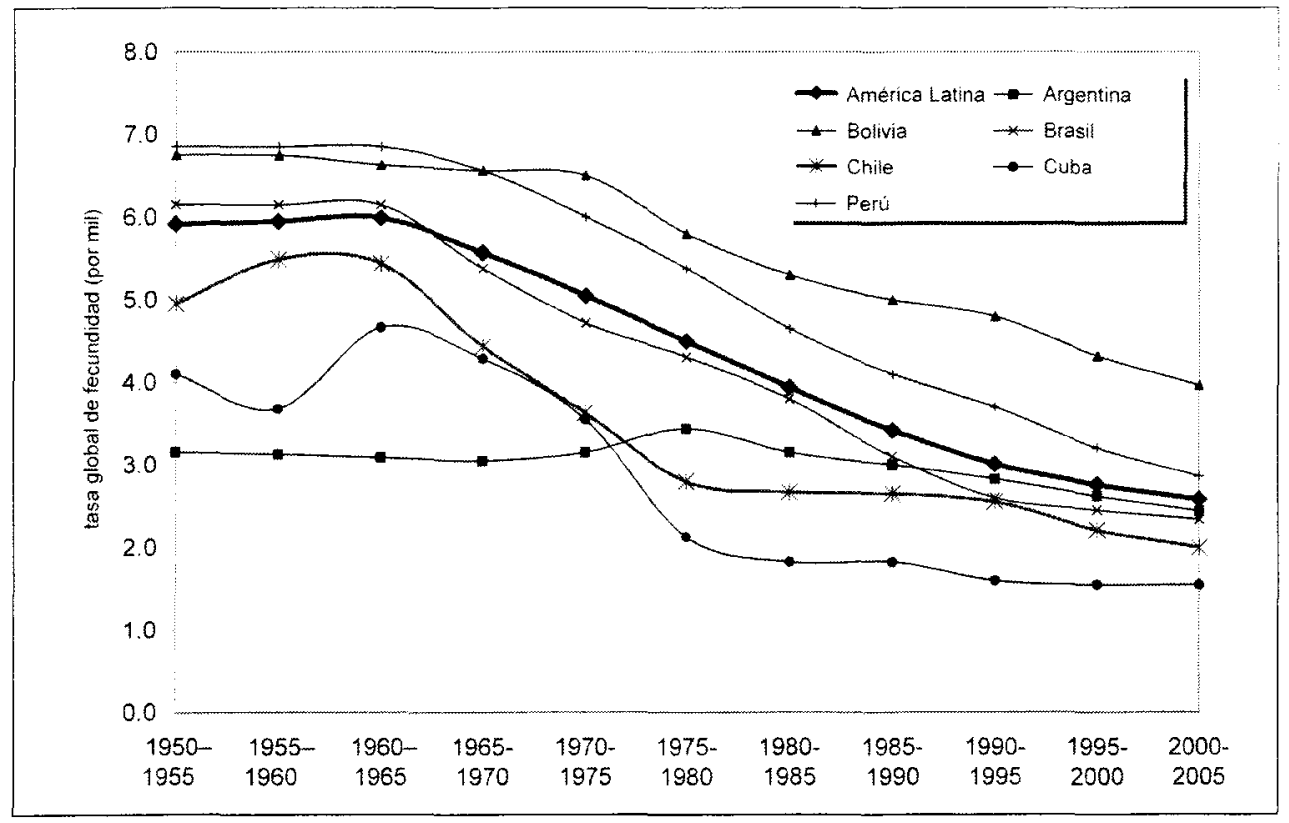

Fuente: Banco de datos del CELADE.

14 Un hecho social de gran importancia es que, en abierto desafio a la posición pronatalista adoptada por el gobierno chileno a mediados de los años setenta æque conllevó la desarticulación de los programas públicos de planificación familiaræ, la fecundidad continuó disminuyendo.

15 Para asegurar el reemplazo de la población se requiere que la TGF sea igual o superior a 2,1, cifra que, en promedio, significa al menos una hija por cada mujer en edad reproductiva. 
2.6. Desigual acceso a los derechos reproductivos. Es sabido que la transición de la fecundidad en América Latina comenzó en las zonas urbanas, entre las mujeres con mayor nivel de instrucción, especialmente aquellas que iniciaron sus uniones maritales después de los 20 años de edad, fenómeno más frecuente en los estratos medios y altos. Fue en estos contextos donde el comportamiento reproductivo experimentó sus primeros descensos, y fue también en ellos donde la decisión de reducir el tamaño de la descendencia pudo materializarse primero. A raíz de esta situación, los regímenes reproductivos urbanos y rurales cobraron diferencias importantes; las especificidades de unos y otros se han mantenido con el paso del tiempo, aun cuando la magnitud de las brechas varía entre los países de la región. Por ejemplo, en los años noventa, las mujeres urbanas de Chile y Cuba tenían, en promedio, un hijo menos que sus congéneres rurales; en cambio, en Honduras y Paraguay, la discrepancia era de tres a cuatro hijos. La brecha urbano rural es mayor en los países menos avanzados en la TD y con menor grado de urbanización, donde la pobreza y el aislamiento se asocian a altos niveles de fecundidad y mortalidad infantil.

Algo similar puede decirse respecto de las brechas según el grado de educación; en general, las mujeres con un mayor grado de educación suelen tener valores que favorecen aspiraciones para sí mismas y sus hijos e hijas y que, por lo común, pueden atenderse mejor en un contexto de familias de tamaño más reducido. En definitiva, la inequidad social, rasgo persistente y significativo de la historia regional, es un factor clave en el ejercicio de los derechos reproductivos.

En todos los países para los que se cuenta con información adecuada (no es el caso de Chile), se verifica un aumento del uso de anticonceptivos desde fines del decenio de 1980 (CEPAL/CELADE, 2002). Ello refleja el deseo creciente de las parejas e individuos de tener familias más pequeñas y de elegir el momento en que tendrán sus hijos (gráfico 10). No obstante lo dicho, el uso de anticonceptivos es claramente mayor entre las mujeres que residen en las áreas urbanas, y la frecuencia de su empleo guarda una relación inversa con el nivel de instrucción de las mujeres. Por ello, la fecundidad no deseada sigue siendo un problema real para las mujeres pobres, rurales y con bajo nivel de educación. Estas diferencias dejan en claro que los estratos sociales más vulnerables son los más desprotegidos en cuanto al ejercicio de sus derechos reproductivos. Sin perjuicio de ello, la información disponible deja en claro que la incidencia del riesgo de una fecundidad alta, que impone una pesada exigencia de crianza a las personas y que suele entrañar el encasillamiento doméstico de las mujeres, es menor en los países donde la transición demográfica se encuentra más avanzada. 


\section{Gráfico 10}

América Latina (países seleccionados): prevalencia anticonceptiva de mujeres en unión según nivel de instrucción, circa 1990

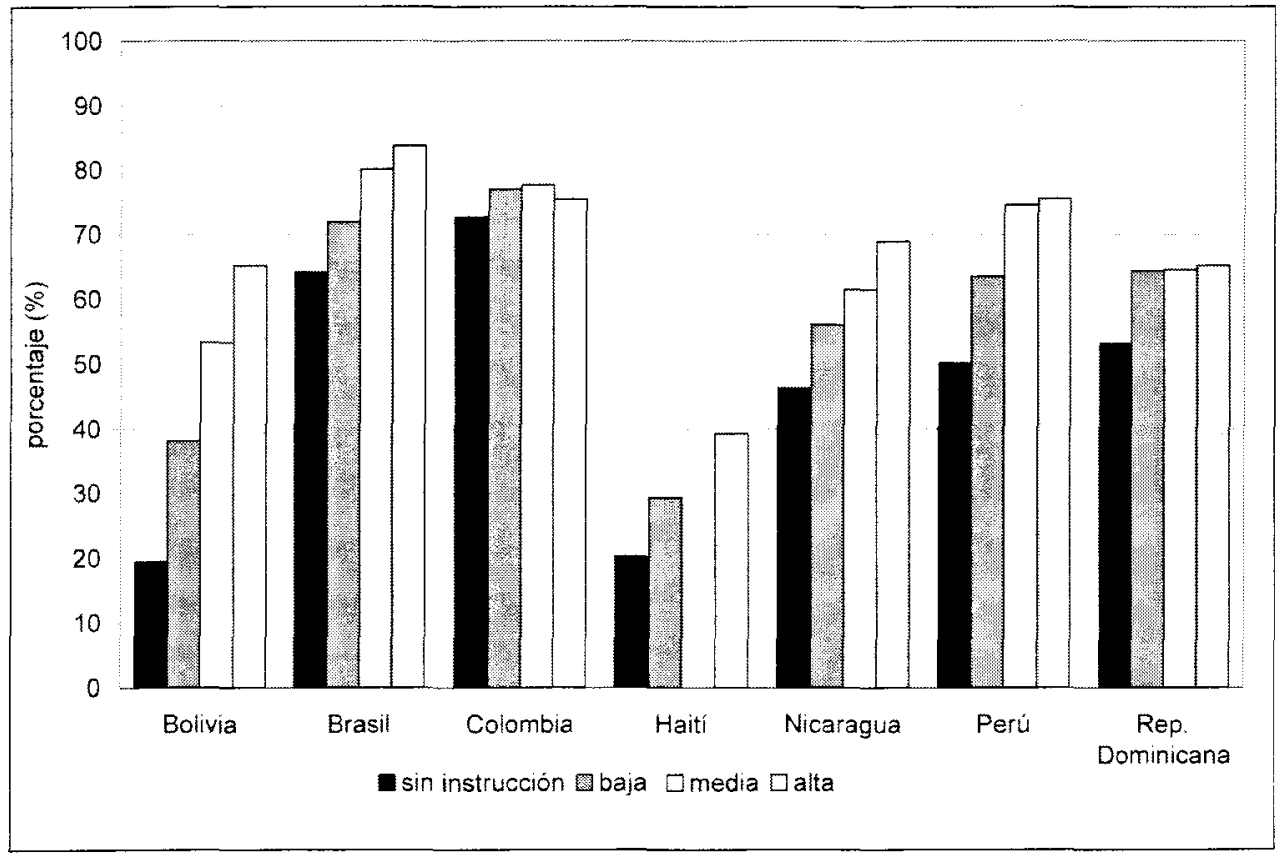

Fuente: $C E L A D E$, Sistema Regional de Indicadores para el seguimiento de conferencias internacionales en América Latina y el Caribe.

2.7. La rebeldía de la fecundidad adolescente. La fecundidad se ha reducido en todas las edades del ciclo reproductivo, aunque lo ha hecho de manera diferenciada. ${ }^{16}$ Durante el último medio siglo, las tasas de fecundidad, según la edad de la mayoría de los países latinoamericanos, se rejuvenecieron, puesto que disminuyó la intensidad reproductiva de las mujeres de $30 \mathrm{y}$ más años de edad. El descenso fue mucho menor entre las adolescentes ( 15 a 19 años), quienes elevaron su contribución relativa al total de los nacimientos. Si bien la fecundidad en la adolescencia es independiente de la transición demográfica, su incidencia se ha hecho más notoria con el avance de este proceso (gráfico 11). Así, en Argentina, la proporción del total de los nacimientos imputables a las adolescentes se incrementó entre 1950-55 y 1975-80, fenómeno asociado al ligero ascenso de la TGF en ese período, pero, posteriormente, se mantuvo estable. En Perú y Bolivia, el peso relativo de las tasas

16 Estas diferencias corresponden a reajustes del calendario reproductivo, que resultan principalmente de la naturaleza de los cambios en los patrones de nupcialidad (adelantamiento o atraso de la edad de ingreso a la unión) y de la estrategia de planificación familiar utilizada (enterar el número deseado de hijos a corto plazo o postergar la procreación, total o parcial, hasta una instancia posterior del ciclo de vida). 
específicas de las adolescentes ha sido menor, si bien en el caso boliviano se produjo un aumento en años recientes. Según la información disponible para Brasil, el porcentaje de nacimientos correspondiente a las adolescentes se duplicó en el último cuarto de siglo, alcanzando al 20\%. Los datos referidos a 1950-55 permiten notar que el nivel de la fecundidad adolescente era similar en Chile y Cuba, pero la evolución posterior revela historias diferentes, aunque ambas implican ascensos de su peso relativo. A raíz del aumento de las tasas específicas, el porcentaje de nacimientos tenidos por las jóvenes cubanas menores de 19 años, se triplicó entre 1950-55 y 1970-75; aun cuando más tarde hubo una ligera atenuación, uno de cada cuatro nacimientos ocurridos en el primer quinquenio del siglo XXI corresponde a las adolescentes. En Chile el incremento ha sido sostenido, pero menos marcado; con todo, a comienzos del siglo XXI las adolescentes dan a luz casi un quinto del total de los nacimientos.

\section{Gráfico 11}

Chile y otros países: Porcentaje de la fecundidad adolescente respecto de la fecundidad total, 1950-2005

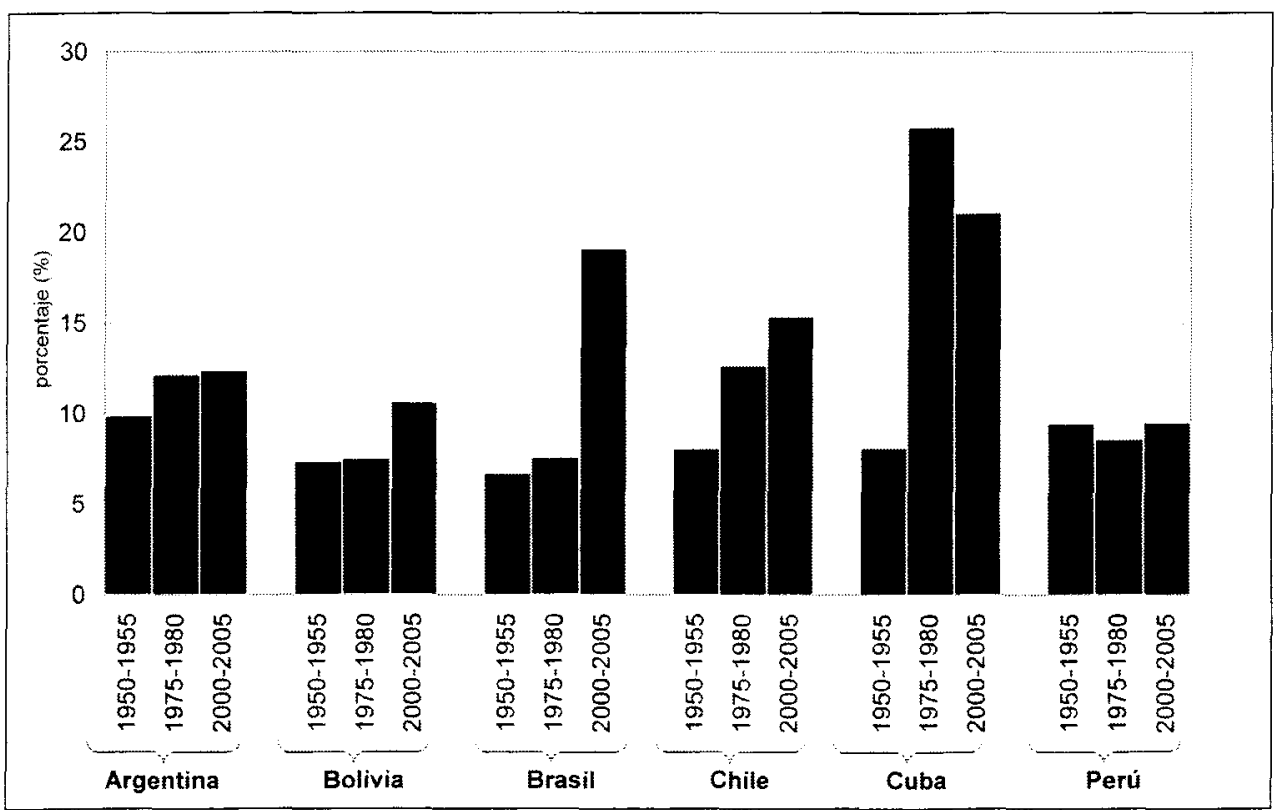

Fuente: Banco de datos del CELADE.

Buena parte de la fecundidad de las adolescentes se inscribe en trayectorias reproductivas intensas, propias de contextos nacionales y subnacionales con rezagos económicos y sociales, donde la tríada de iniciaciones (sexual, nupcial y reproductiva) se verifica a temprana edad y en intervalos reducidos, configurando un modelo tradicional que restringe el papel de las jóvenes al ámbito doméstico. Otra parte de la fecundidad de las adolescentes se manifiesta en contextos de mayor avance 
socioeconómico y de la transición demográfica, donde las exigencias sociales colisionan con la unión y la reproducción tempranas, originando una incompatibilidad entre el papel asignado a muchachas y muchachos, y las obligaciones de la pareja y la crianza; si bien esta incompatibilidad no se aplica a la esfera sexual, ya que muchos adolescentes inician su actividad sexual a edad temprana, fuera de uniones; una alta proporción, ya sea por razones culturales, familiares o institucionales, no accede a medios anticonceptivos o no los usa adecuadamente, lo que deriva en altas tasas de fecundidad temprana, por lo común no deseada, y configura un modelo de modernidad sexual y reproductiva truncada.

Una medida no convencional, basada en la proporción de adolescentes que eran madres a la fecha del censo, ilustra la tendencia de la fecundidad adolescente en países ubicados en diferentes instancias de la transición demográfica (gráfico 12). No puede menos que llamar la atención que un cuarto o más de las muchachas que aún no enteraban los 20 años ya era madre; esta proporción aumentó, en la mayoría de los casos, durante el decenio de 1990. El caso de Chile, que aparece como una excepción a raíz de la ligera disminución de aquel porcentaje, presenta otra faceta no menos inquietante: el incremento de la maternidad entre las niñas de 15 y 16 años, fenómeno que también se manifiesta en Uruguay y en Brasil.

\section{Gráfico 12}

Chile y otros países: porcentaje de mujeres adolescentes que son madres según

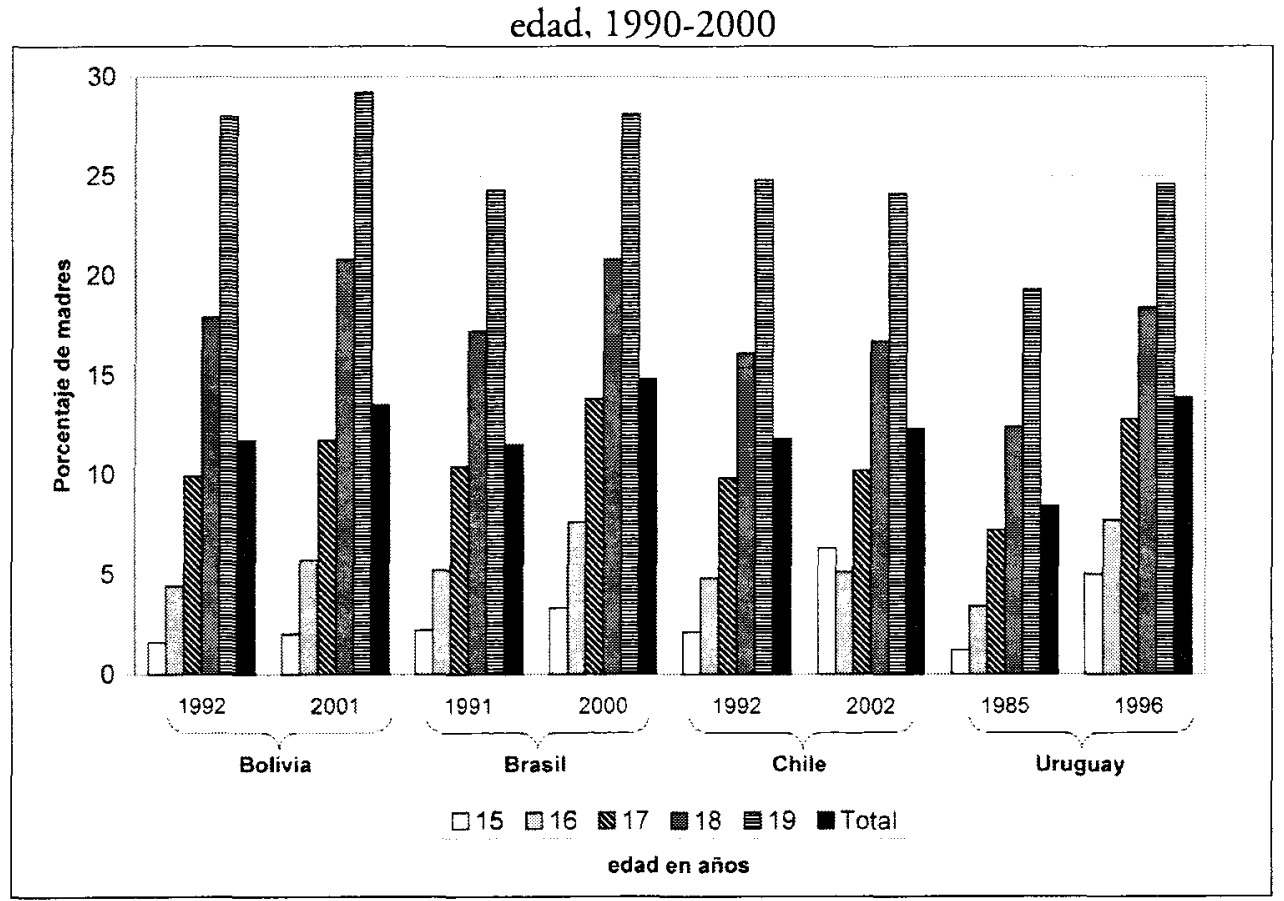


La elevada fecundidad adolescente es también un componente de la dinámica demográfica de la pobreza. Ello origina una forma de adaptación resignada, que se expresa en el ingreso temprano de los y las jóvenes a la fuerza de trabajo en desmedro de su formación (CEPAL/CELADE, 2002). En Chile y Brasil se observa un dramático contraste entre el tipo de actividad que desarrollan las adolescentes que han sido madres y aquéllas que no han tenido esta experiencia (gráfico 13). La mayoría de las muchachas que no son madres continúa en el sistema escolar; más aún, durante el decenio de 1990 disminuyó la proporción de aquéllas que están insertas en la población económicamente activa (PEA) y en las labores domésticas. Por el contrario, sólo una fracción pequeña de las que son madres, que apenas llega a un quinto en Chile en 2002 (después que fuera menos de un décimo en 1992), sigue estudiando; la mayoría, en cambio, desarrolla faenas del hogar (no estudian ni hacen parte de la PEA).

\section{Gráfico 13}

Chile y Brasil: tipo de actividad económica de las mujeres adolescentes según condición de maternidad, 1990-2000

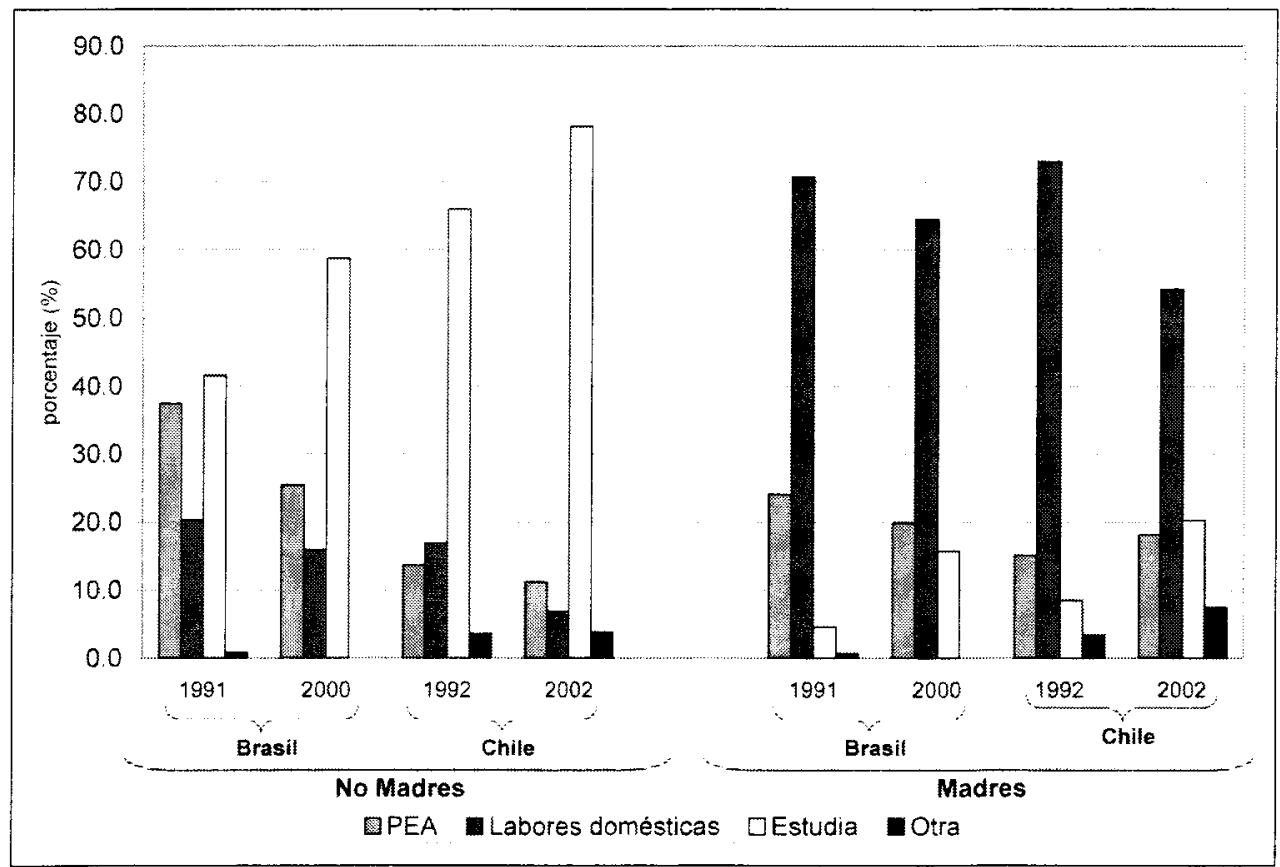

Fuente: Banco de datos del CELADE.

El uso de anticonceptivos en la primera relación sexual de las adolescentes es muy reducido en los países de la región para los que se dispone de información (no es el caso de Chile). Todo parece indicar que existe un rezago de los programas de educación sexual y salud reproductiva orientados a la adolescencia, lo que contribuye a entender la persistencia de los altos niveles de fecundidad temprana en la región. La comparación entre Brasil y España es aleccionadora: en ambospaíses se 
registra una prevalencia similar de anticoncepción entre las mujeres de más de 20 años; sin embargo, en Brasil esta prevalencia se reduce a menos de un tercio de las adolescentes y en España está por encima del $80 \%$. Si bien la edad mediana de inicio de las relaciones sexuales es similar en ambos países, la fecundidad en la adolescencia es mucho mayor en Brasil, lo que brinda un indicio de modernidad sexual y reproductiva truncada, que se hace ostensible a raíz de que la fecundidad brasileña está descendiendo intensamente.

2.8. ¿Qué ocurrirá con la fecundidad en el futuro? La evolución futura de la fecundidad en la región es un asunto incierto, que motiva diversas especulaciones e interrogantes. ${ }^{17}$ Una de ellas es si su descenso seguirá las líneas del caso de Cuba, alcanzando un nivel similar al de los países del sur de Europa o si, por el contrario, se irá frenando gradualmente, sin traspasar el umbral del reemplazo, como hasta ahora ha ocurrido en Argentina y Uruguay. Los antecedentes que brindan los censos de la ronda de 2000 y las últimas versiones de las encuestas especializadas, indican que aquel umbral está muy cerca en varios países; Chile, en particular, lo acaba de alcanzar. Con todo, estas mismas fuentes entregan indicios de una barrera: la profunda y persistente desigualdad social asociada al rezago de los grupos sociales más desfavorecidos, que enfrentan serias dificultades para ejercer sus derechos básicos a la vida y sus derechos reproductivos y sexuales (Chackiel y Schkolnik, 2004; CELADE, 2004).

No obstante lo señalado, el ejemplo de Chile, al que es probable que se agregue Brasil, a corto plazo, junto a México y Colombia, en un futuro no lejano, sugiere que la fecundidad puede llegar a niveles bastante reducidos, merced a la generalización de su control entre los diversos estratos, sin que se cumpla la condición de una mayor equidad social, rasgo que sí ha estado presente, en distinto grado, en las experiencias de Cuba, Uruguay, Costa Rica y Argentina. Todo ello parece abonar la tesis según la cual el campo demográfico ha adquirido un creciente grado de autonomía respecto de sus bases materiales, aunque quizás no de sus determinantes culturales.

\section{La región se envejece.}

3.1. La progresiva remodelación de la pirámide de edades. Casi todos los fenómenos sociales son influenciados por la estructura etaria de la población, antecedente fundamental para la programación de los servicios sociales, la estimación y proyección de las demandas de bienes y servicios, y del potencial de trabajo de una sociedad. Durante la mayor parte de su historia, la población de América Latina se

17 Estas interrogantes animaron los debates del seminario "La fecundidad en América Latina y el Caribe: ¿transición o revolución?", que el CELADE celebró en junio de 2003; los documentos presentados, actualmente en prensa, están disponibles en www.eclac.cl. Cabe añadir que las últimas proyecciones de población preparadas por el CELADE incorporan el supuesto æformulado de consuno con la División de Población de las Naciones Unidasæ de que la TGF de la mayoria de los países de América Latina será de 1,86 hijos por mujer en el año 2050; según aquellas proyecciones, este valor se alcanzaría en Chile antes de 2020 (CEPAL, 2004a). 
ha distinguido por una estructura juvenil. La caída de la mortalidad, que redundó en una mayor supervivencia infantil, contribuyó a que este rasgo de mocedad se mantuviera hasta después de iniciada la declinación de la fecundidad. La población menor de 15 años representaba el 40\% del total de efectivos de la región en 1950, cuando la relación de dependencia era de 78 personas en edades inactivas por cada 100 potencialmente activos. ${ }^{18}$ La mayoría de los países se encontraba en una etapa incipiente o moderada de transición y la distribución de su población según edad mostraba el perfil nítido de una pirámide regular, de amplia base (gráfico 14). En los países que tenían una transición demográfica avanzada en 1950, Uruguay, Argentina $y$, en menor medida, Cuba, la pirámide era menos esbelta y sus estratos inferiores eran bastante menos dilatados. Una silueta intermedia exhibía la estructura por edades de la población chilena, en la que los menores de 15 años no alcanzaban a enterar el $37 \%$ del total de los habitantes del país.

\section{Gráfico 14}

Chile y otros países en transición demográfica avanzada y moderada: Estructura por edad y sexo, 1950-2000

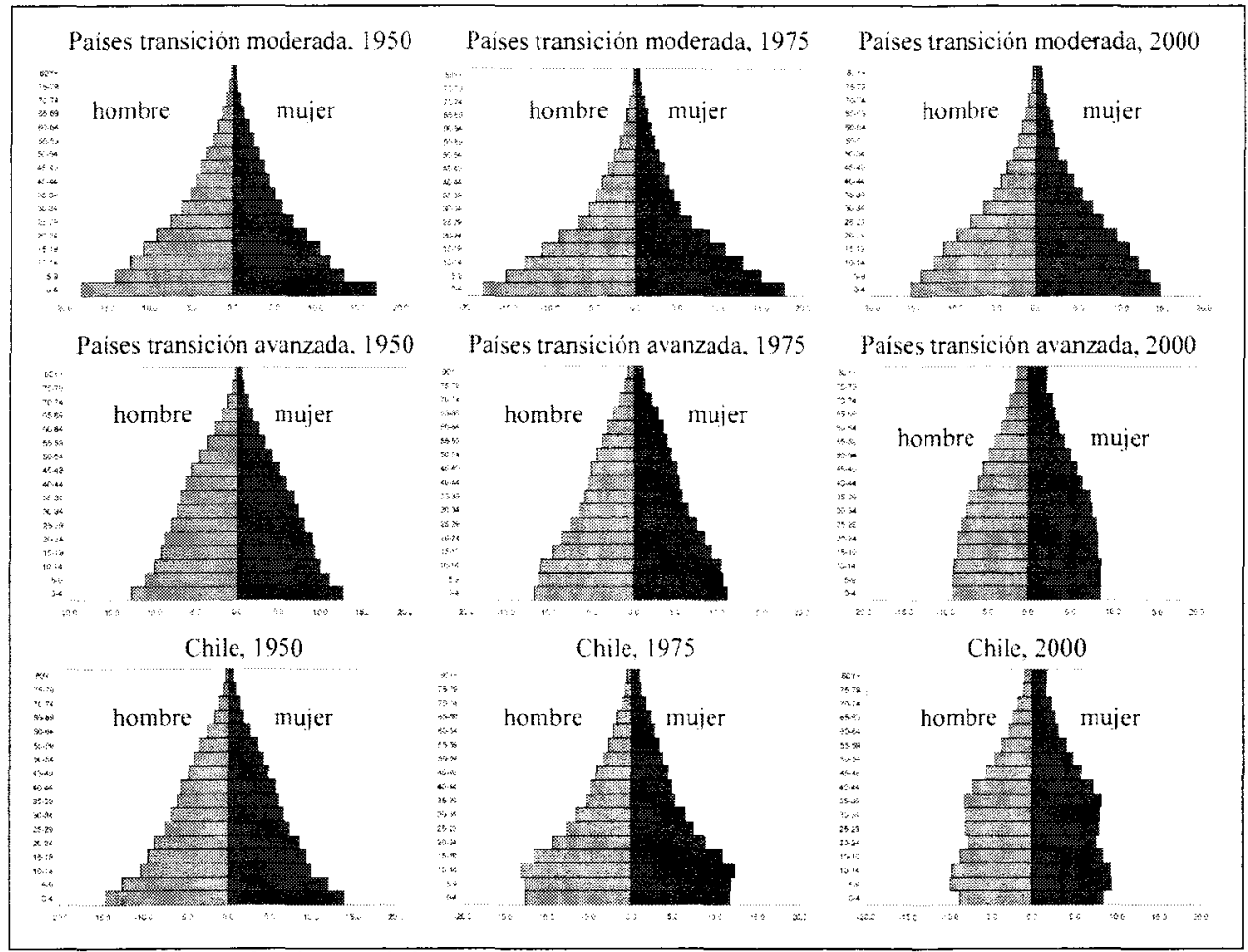

Fuente: Banco de datos del CELADE.

18 La relación entre la población joven y adulta mayor con respecto a la población adulta (en edad de trabajar) se llama relación de dependencia demográfica o de la edad. Su cálculo convencional se realiza mediante el cociente entre la suma de las personas menores de 15 años y mayores de 60 , y la de 15 a 60 . 
Durante los años sesenta, a raíz del fuerte descenso de la mortalidad y de una fecundidad que se mantenía elevada, los menores de 15 años representaron el $43 \%$ de la población de América Latina, pero su peso relativo excedía del $46 \%$ en diez países; la relación de dependencia aumentó a $89 \%$ en el conjunto de la región. Este rejuvenecimiento también se observó en Chile, donde los menores de 15 años llegaron a constituir el $40 \%$ de la población total. El descenso de la fecundidad, que comenzó a mediados de los años sesenta, dio la partida a una tendencia opuesta, que redundó en el gradual angostamiento de la base de la pirámide de edades. Este efecto se hizo notorio en los países de transición demográfica avanzada a mitad del decenio de 1970 , pero aún no se advertía con nitidez en aquéllos que estaban en etapas moderadas o incipientes del proceso, puesto que el factor de inercia demográfico, asociado a la alta frecuencia de nacimientos de la densa cohorte de mujeres nacidas entre 15 y 25 años antes, aseguraba el vigor del crecimiento demográfico y se traducía en una pirámide de amplia base. ${ }^{51}$ En cambio, la estructura etaria de la población de Chile ya daba señales de una madurez relativa, con una proporción de niños menores de 15 años que empezaba a disminuir.

En el año 2000 los menores de 15 años eran un tercio de la población regional y la relación de dependencia se redujo al $60 \%$. Debido principalmente al descenso de la fecundidad, la proporción de efectivos en los tramos superiores de edad se ha venido acrecentando a paso firme. Los latinoamericanos nacidos durante la era de multiplicación acelerada de la población, a mediados del siglo XX, comenzarán a incorporarse a los escalones superiores de la pirámide en 2015, dando lugar a una era de retiro que se prolongará en los decenios siguientes. El número de adultos mayores se duplicará con creces entre 2000 y 2025 , aumentando de 27 a 66 millones de personas, y volverá a duplicarse entre 2025 y 2050 para llegar a 136 millones, casi un quinto de la población regional. La velocidad de este proceso de envejecimiento guarda directa relación con la rapidez del descenso de la fecundidad. En suma, la pirámide de edades de la población ya empezó a perder su tradicional perfil triangular y se acerca, cada vez más, a otro de forma rectangular.

19 La importancia del factor de inercia demográfica estriba en que la estructura por edades de un momento dado opera como un tamiz de los cambios subsecuentes de la mortalidad y la fecundidad, difiriendo los impactos modeladores de estos cambios en aquella estructura. Por ello es que los efectos del descenso de la fecundidad en la segunda mitad de los años sesenta se vieron transitoriamente diluidos a raíz del aumento en el tamaño de las cohortes de mujeres en edad fértil (fenómeno resultante de la transferencia del mayor ritmo de crecimiento demográfico en los decenios anteriores). 
3.2. El inexorable proceso de envejecimiento. El envejecimiento suele describirse como el incremento sostenido de la proporción de personas de 60 y más años con respecto a la población total. Este fenómeno resulta de la progresiva alteración del perfil de la estructura por edades, cuyos rasgos piramidales "clásicos", con una base amplia y una cúspide angosta, se van desdibujando para adquirir una fisonomía rectangular y tender, posteriormente, a la inversión de su forma inicial (Chesnais, 1990). Esta secuencia, a diferencia de lo que ocurre con el envejecimiento de los individuos, no sigue un ritmo cronológico único, puesto que, además del factor de inercia inherente a toda composición etaria, obedece al sentido, fuerza y persistencia de los cambios de las variables demográficas fundamentales: mortalidad, fecundidad y migración.

La disminución de la mortalidad y el concomitante aumento de la esperanza de vida, conducen a que más personas sobrevivan hasta edades avanzadas. Si bien esto implica la ampliación del tamaño de las cohortes que irán avanzando por los sucesivos peldaños de la pirámide de edades, el descenso de la mortalidad no siempre impulsa el envejecimiento de la población. Así, la transición hacia menores niveles de mortalidad se inició en América Latina con una reducción más acentuada de las muertes en la infancia y la niñez temprana, redundando en un rejuvenecimiento de la población, un efecto similar al que se desprendería de un aumento de la fecundidad. Tal sesgo se asoció con la adopción de tecnologías médicas y programas de atención de la salud de bajo costo y de aplicación relativamente fácil. Sólo después de haber conseguido progresos importantes en las etapas iniciales de la vida, las intervenciones dirigidas a aminorar la mortalidad comenzaron a distribuirse de manera más equilibrada entre las distintas edades, por lo que sus repercusiones sobre la estructura por edades no fueron mayores. En años recientes, y en asociación con el proceso de transición epidemiológica, los programas de atención de la salud han contribuido a que la reducción de la mortalidad rinda frutos entre los adultos mayores; así, el aumento de la probabilidad de sobrevivir después de los 60 años impulsa, de modo directo, el envejecimiento de la población. ${ }^{20}$

20 En América Latina, el descenso de la mortalidad infantil ha sido mucho más intenso que el observado entre la población adulta y, en particular, que en las edades avanzadas. Un indicio de esta diferencia se obtiene de la comparación de las ganancias de la esperanza de vida de la población total con las conseguidas por el grupo de 60 y más años: casi un $80 \%$ del aumento del valor del indicador regional entre 1950 y 2000 correspondió al incremento de la probabilidad de sobrevivir hasta los 60 años (Villa y Rivadeneira, 2000). Chackiel y Plaut (1994), mediante un modelo basado en el comportamiento histórico de la mortalidad de Costa Rica y Cuba, muestran que la mayor disminución, en términos absolutos y relativos, corresponde a las edades más bajas: cuando la esperanza de vida aumenta de 50 a 75 años, el riesgo de morir de los niños menores se reduce en $90 \%$ y el de las personas de 65 y más años en $34 \%$. 
Sin embargo, la fecundidad es la principal fuerza modeladora de la estructura por edades de la población; por ende, y aunque choque al sentido común, sus modificaciones provocan un impacto mayor y más directo sobre el envejecimiento que los cambios de la mortalidad, por lo menos hasta que se logren mayores progresos en la reducción de la mortalidad en edades adultas y avanzadas. Como apunta Chesnais (1992 y 1990), el resultado inmediato del acentuado descenso de la fecundidad es el "envejecimiento por la base", es decir, la disminución de la proporción de niños respecto de la población total. La persistencia de este descenso por un tiempo prolongado llevará al "envejecimiento por el centro" (una figura rectangular); posteriormente, y en la medida que su efecto se combine con el de la disminución de la mortalidad en las edades avanzadas, originará estructuras etarias con una cúspide amplia y una base estrecha (tal vez con forma de hongo). De este modo, la transición hacia menores niveles de fecundidad actúa como detonador de la "inversión de la pirámide". La comparación de las pirámides de edad de los países latinoamericanos en distintas instancias de la transición demográfica, permite apreciar estos efectos.

A raíz de su indisoluble vínculo con la transición demográfica, el envejecimiento alcanza niveles y trayectorias heterogéneos entre los países de la región (CEPAL, 2000; Villa y Rivadeneira, 2000). Los más avanzados en la transición, en especial los que iniciaron antes este proceso, son los más envejecidos (gráfico 15); así, en Argentina las personas mayores representaban el 13\% de la población en 2000, proporción que casi duplica la existente en 1950 , aunque cabe advertir que la tasa de crecimiento de este grupo etario ha venido declinando en los últimos años. La acelerada transición en Cuba ha dado lugar a un ascenso más rápido, ya que en 2000 esa proporción fue del 14\% y se estima que un cuarto de la población en 2025 será adulta mayor. Bolivia y Perú registraron en 2000 porcentajes equivalentes a la mitad de los observados en Argentina y Cuba, respectivamente, pero sus proyecciones indican un rápido incremento en los años futuros. En Brasil, la tasa de crecimiento de la población adulta mayor se ha mantenido elevada y su actual porcentaje dentro del total (casi 8\%), se duplicará en 2025. La condición de Chile es intermedia, pues las personas mayores representaron poco más del $10 \%$ de la población en 2000, aunque el ritmo de incremento de ese conjunto hace esperar que en 2025 se constituya en un quinto del total de efectivos. 


\section{Gráfico 15}

Chile y otros países: porcentaje de población adulta mayor y tasa de crecimiento, $1950-2000$

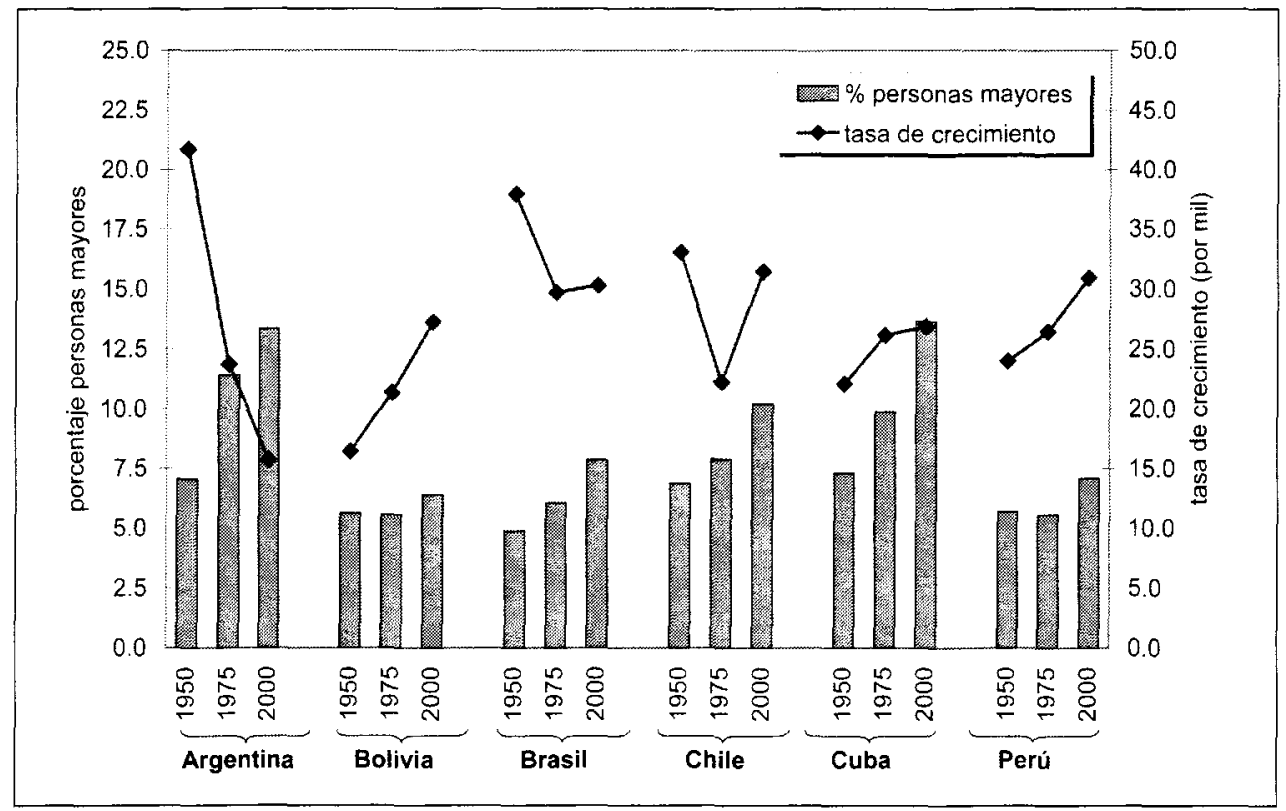

Fuente: Banco de datos del CELADE.

Entre los efectos demográficos directos del proceso de envejecimiento, se destaca la alteración de la relación proporcional entre las generaciones. Una manera de evaluar este impacto consiste en estimar el índice de envejecimiento, que expresa el número de personas de 60 y más años de edad por cada 100 menores de 15 años (gráfico 16). A raíz del mayor ritmo de crecimiento de los adultos mayores no sorprende que los valores de este índice sean ascendentes en todos los países. El incremento más notable se aprecia en Cuba, ya que se triplicó entre 1950 y 2000; en Argentina, el índice se duplicó en igual lapso. Los indicadores de Bolivia y Perú se mantienen en rangos bajos, ya que en ambos países las personas mayores no llegaron a ser equivalentes a un cuarto del total de niños en 2000; Brasil, que tuvo cifras similares a Bolivia y Perú en 1950, experimentó un aumento notable en los últimos decenios y en 2000 se contabilizaron casi 30 adultos mayores por cada 100 personas menores de 15 años. En el caso de Chile se aprecia una situación intermedia, si bien el valor del índice virtualmente se duplicó en el último cuarto del siglo XX.

Una característica distintiva del envejecimiento es su especificidad de género; en general, a medida que aumenta la edad de una población se acrecienta la proporción de mujeres y disminuye el índice de masculinidad. En casi todos los países de América Latina y el Caribe, y a lo largo del período 1950-2000, la población adulta 
mayor presenta este signo distintivo. El sistemático predominio numérico femenino es aún mayor en el subconjunto de la cuarta edad. Esta especificidad se origina en la mortalidad diferencial según sexo, que redunda en una mayor esperanza de vida las mujeres; la longevidad más prolongada de las mujeres se acentúa a medida que desciende el nivel general de la mortalidad. En promedio, las mujeres viven seis años más que los hombres en la región, y a la edad de ingreso a la adultez mayor, la diferencia es de casi cuatro años. Estas condiciones han ido variando con el avance de la transición demográfica, como se deduce de la gradual disminución de la relación de masculinidad en Cuba, Argentina y Chile.

\section{Gráfico 16}

Chile y otros países: índice de envejecimiento y relación de masculinidad de la población adulta mayor, 1950-2000

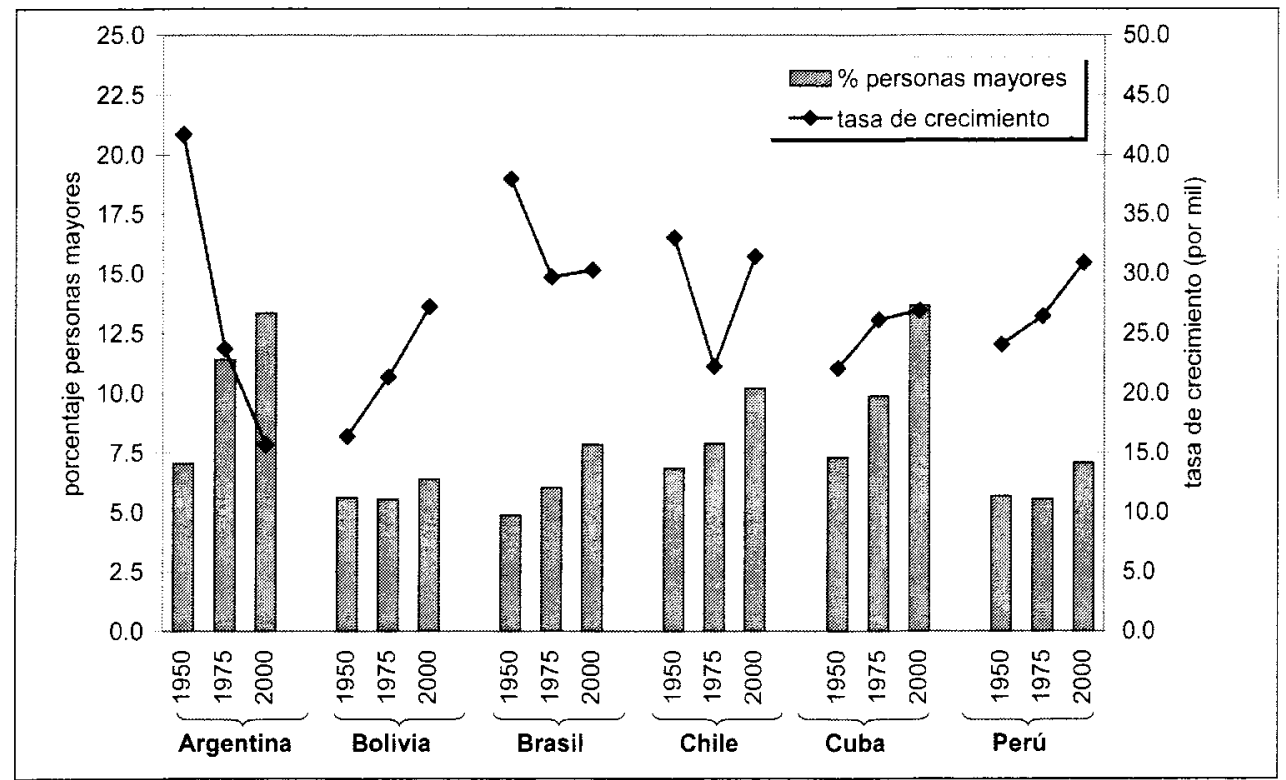

Fuente: Banco de datos del CELADE.

Sin lugar a dudas, más importante que la mera longevidad es la calidad de los años que se viven. Según se desprende de la información de los censos de varios países latinoamericanos, entre un cuarto y un tercio de las mujeres de 60 a 64 años no tienen cónyuge, ya sea porque enviudaron o porque se mantuvieron célibes; esta proporción se eleva a más de la mitad entre las personas de 75 años y más. La ausencia de una pareja, amén de restringir las posibilidades de enfrentar las necesidades básicas, crea carencias afectivas y repercute sobre la seguridad emocional. La frecuencia más elevada de la viudez entre las mujeres no sólo obedece a la diferencia de la esperanza de vida, sino también a los efectos de las pautas culturales asociadas 
a los patrones de nupcialidad (los hombres se unen con mujeres de menor edad), y a la mayor probabilidad de que los hombres establezcan una nueva unión tras la viudez o la ruptura de una relación anterior. Hay otras razones por las que la ventaja de la mayor longevidad de las mujeres resulta más aparente que real, ya que las inequidades de género sufridas a lo largo de la vida se acumulan en las edades avanzadas y hacen extremadamente difícil esta etapa de la existencia. Es sabido que el trabajo de la mujer no siempre recibe reconocimiento formal y que el ingreso que obtiene a cambio de su esfuerzo, corrientemente, es inferior al percibido por el hombre que realiza actividades similares. Esta condición desmedrada de la mujer limita sus posibilidades de generar ahorro para la adultez mayor y tiende a marginarla de las transferencias de recursos de los sistemas de previsión social. Todas estas circunstancias se suman a la soledad de muchas mujeres de edad, sea a raíz de la viudez o de la soltería.

3.3. Los viejos ya no tienen tiempo para esperar. El envejecimiento de la población, generado por la transición demográfica, repercute sobre las variables económicas y financieras (demanda, ahorro, competitividad, inversión en salud y seguridad social), el perfil epidemiológico y la estructura social. Algunas de estas consecuencias son previsibles y otras hipotéticas, por lo que dan lugar a incertidumbres. Como el envejecimiento no es, en sí, un proceso evitable en términos reales, el reto consiste en buscar los medios que permitan enfrentar sus adversidades. Desde este punto de vista cabe apuntar que es probable que la población de América Latina sea la primera región no desarrollada que envejece. En la actualidad, y más allá de la heterogeneidad de trayectorias de la transición demográfica, el envejecimiento de la población regional se encuentra en un punto de inflexión a partir del cual se acelerará intensamente. De allí la oportunidad histórica del momento actual para que los países adopten medidas que fortalezcan su capacidad de respuesta a los efectos económicos, sociales e institucionales que el proceso trae consigo; también es este el momento para propiciar mecanismos de adaptación activa, propios de "una sociedad para todas las edades".

Se entiende que una sociedad para todas las edades es aquélla que, guiada por los principios de reciprocidad y equidad, ajusta sus estructuras y funcionamiento, sus políticas y programas, a las necesidades y capacidades de todos, de modo que las generaciones puedan efectuar inversiones recíprocas y compartir los frutos de esas inversiones. Por ende, si los países de América Latina encuentran un camino de progreso y justicia, las personas de edad no podrán quedar excluidas. La pregunta clave es cómo ayudar a que este grupo etario participe plenamente en lo que el progreso posibilita y la justicia exige. Todo el tema de los sistemas previsionales y de jubilación está centrado en este aspecto, como también lo están la salud, la vivien- 
da, la organización de la familia y la comunidad, el tiempo libre e, incluso, la educación de las personas de edad. En términos más generales, la inserción social de estas personas está relacionada con la equidad.

Es importante destacar que el grado y la evolución del envejecimiento æcomo resultado de las dispares trayectorias del descenso de la fecundidad y la mortalidad de los diversos estratos socioeconómicos y territorialesæ también presentan diferencias dentro de los países. Los grupos que se encuentran en mejores condiciones socioeconómicas son los que presentan un mayor grado de envejecimiento, pero a medida que se vaya generalizando la caída de la fecundidad y la mortalidad, disminuirán las discrepancias entre las estructuras etarias de los distintos estratos socioeconómicos y el envejecimiento ya no será resultado de una suerte de "selección natural". Dado que este proceso es todavía incipiente en la región, sus consecuencias adversas aún no se manifiestan con suficiente nitidez en las cifras agregadas; sin embargo, en los países más avanzados en la transición demográfica (Argentina, Uruguay, Chile y Cuba) se observan algunos de sus efectos adversos. Entre ellos se destaca el aumento sostenido de la carga demográfica en los sistemas de pensiones (relación entre jubilados y cotizantes), que se expresa en una creciente presión financiera y sirve de aliciente a las iniciativas a favor del reemplazo total o parcial de los mecanismos de reparto æbasados en el principio de solidaridad intergeneracionalæ por otros que descansan en la capitalización individual, cuyos resultados, aún inciertos, son objeto de amplio debate.

Los cambios en la estructura por edades de la población guardan íntima relación con la evolución de los perfiles epidemiológicos, pues los patrones de morbilidad transitan de una situación en que predominan enfermedades infecciosas y agudas a otra en que prevalecen las crónicas y degenerativas, propias de las edades avanzadas. Sin embargo, las diferencias en las causas de muerte entre los países, y dentro de los mismos, ponen de manifiesto la persistencia de enfermedades ligadas al subdesarrollo y la pobreza. Esto exige impulsar estrategias de salud que contemplen la atención prioritaria de los grupos sociales vulnerables. Con relación a los adultos mayores, es sabido que los costos de atención de su salud superan a los de otras edades, y que la capacidad instalada de las instituciones es actualmente insuficiente para atender sus demandas específicas. De allí la necesidad de establecer sistemas de salud integrados, basados en una atención primaria descentralizada y participativa, que estimule el auto-cuidado, la atención informal y la participación de la familia y la comunidad. En tal sentido, la promoción de una esperanza de vida en buena salud implica prevenir, retardar o disminuir los problemas que puedan afectar la capacidad de los individuos para actuar por sí mismos. 
Históricamente, la familia ha sido concebida como la unidad social y biológica que, además de proveer sustento y socialización a las nuevas generaciones, debe brindar apoyo emocional, económico, social y de salud a sus miembros de mayor edad. En América Latina y el Caribe, la falta de una tradición institucional y comunitaria de atención a los adultos mayores, convierte a la familia en la entidad responsable de su cuidado e integración social. Las modificaciones estructurales producidas en la región y los cambios sociodemográficos han afectado el significado y el funcionamiento de la familia, repercutiendo en las relaciones entre sus integrantes, particularmente las que se establecen con los miembros adultos mayores. Ello exige encontrar mecanismos que, junto con reforzar el papel de la familia, armonicen la equidad en las relaciones multigeneracionales con la equidad intrageneracional.

\section{Un esbozo de recapitulación.}

La apretada revisión de la dinámica demográfica efectuada en este texto permite indicar que la experiencia de Chile, si bien presenta especificidades, no configura un caso excepcional en América Latina. Su proceso de transición demográfica, desde niveles altos de mortalidad y fecundidad a mediados del siglo XX a bajos al empezar el siglo XXI, fue más rápido que el del promedio regional, pero menos veloz que el registrado por otros países, y se inició más tarde que el de Argentina y Uruguay. Los progresos que Chile ha obtenido en la reducción de la mortalidad son considerables, como lo revela la ganancia de casi 23 años de esperanza de vida entre 1950 y 2005; con todo, varios países han conseguido avances parecidos y Costa Rica ocupa el liderazgo de la región en el primer quinquenio del siglo XXI. Tal vez uno de los logros más importantes de Chile sea la reducción de la tasa de mortalidad infantil, que al comienzo del siglo XXI es un $95 \%$ inferior a la existente en 1950-55; sin embargo, Cuba le aventaja con índices más reducidos. También se advierte que el descenso de la fecundidad ha sido intenso en Chile, pero menos que el de Brasil en los últimos años o el de Costa Rica en los decenios de 1970 y 1980.

A raíz del conjunto de cambios ocurridos, el rostro demográfico de América Latina, como el de Chile, es muy diferente de aquel de hace apenas medio siglo. La etapa aquella de la llamada "explosión demográfica" pertenece al pasado; quedó sepultado el viejo régimen en el que campeaban unos muy intensos ritmos reproductivos y otros no menos elevados de mortalidad. En el nuevo régimen, la anterior abundancia de nińos es trocada por una creciente frecuencia de personas adultas mayores y un estilo de crecimiento cada vez más moderado. Dicho lo anterior, debe recordarse que los promedios, por definición, ocultan dispersiones: la heterogeneidad de situaciones imperantes en la región, relacionadas íntimamente 
con la histórica desigualdad de las condiciones materiales de vida, se expresa en distingos entre países y, en especial, entre estratos socioeconómicos; los derechos conseguidos, y formalmente consagrados, en lo que dice relación con la prolongación de la vida y la procreación de una determinada descendencia, todavía no están al acceso de todos.

Los cambios aludidos están enclavados en profundas transformaciones sociales y culturales, que han dado lugar a que las decisiones en el ámbito de la reproducción se manifiesten en un tamaño medio menor de familia, dentro de la cual la coexistencia de los niños y los abuelos se está haciendo más común. Sin embargo, este sostenido y drástico descenso de la fecundidad no ha ocurrido por igual entre las potenciales madres; no puede menos que inquietar, por sus repercusiones sobre la vida futura, la creciente contribución de las jóvenes adolescentes a la reproducción total. Este fenómeno trasunta las dificultades que tienen los programas de educación y salud sexual y reproductiva para interpretar a las y los jóvenes, pero también sugiere que la distancia entre las expectativas y las realidades que rodean la construcción de planes de vida es considerable, quizás por el hecho de que las oportunidades que les abre el sistema socioeconómico son más bien escasas (recuérdese, por ejemplo, que las tasas de desocupación juvenil duplican los promedios nacionales).

En Chile, en particular, el mayor número de nacimientos anuales del que exista registro, ocurrió en la primera mitad del decenio de 1990; en los años siguientes, incluso los venideros, ese número se mantendrá oscilando en cifras inferiores a las enumeradas en los años cincuenta. También en Chile, y a pesar del descenso radical de la mortalidad y del aumento de la esperanza de vida al nacer, la cantidad de defunciones anuales, que disminuyó hasta comienzos de los años noventa, está comenzando a incrementarse, y se estima que a mediados del siglo XXI se hará similar a la cantidad de nacimientos. Sólo en entonces, cuando los chilenos sean poco más de 20 millones, será probable que el país alcance un crecimiento nulo de la población; en espera de ese momento, el efecto de inercia, herencia de la estructura etaria juvenil construida en el pasado, continuará aportando su potencial implícito de incremento. Por de pronto, la sociedad chilena tiene ante sí el reto de acostumbrarse (adaptarse, pero no resignarse), a que las demandas inherentes al ciclo de vida de las personas y los hogares, se está trasladando a edades propias de la madurez y la adultez mayor. Dicho reto impone una tarea respecto de todas las generaciones, que no sólo vivirán en un mismo tiempo, sino que también compartirán una historia presente. 


\section{Referencias}

ABEP/CELADE/IUSSP/PROLAP/SOMEDE (1994), La transición demográfica en América Latina y el Caribe, Ciudad de México, Instituto Nacional de Estadística, Geografía e Informática (INEGI).

Bajraj, R., M. Villa y J. Rodríguez (2000), Población y desarrollo en América Latina y el Caribe: un desafio para las politicas públicas, CEPAL, Serie Población y Desarrollo, No. 7, agosto

CEPAL (2004a), América Latina y el Caribe: estimaciones y proyecciones de población, 1950-2050, Boletín Demográfico, año 37, No. 73, CELADE/División de Población de la CEPAL, LC/G.2225-P.

(2004b), Una década de desarrollo social en América Latina, 19901999, Santiago de Chile, CEPAL, LC/G.2212-P.

(2000), Equidad, desarrollo y ciudadania, Santiago de Chile, CEPAL, LC/G.2071.

(1984), "Población y desarrollo en América Latina", Notas de Población, No. 34, pp. 9-77.

CEPAL/CELADE (2004), Conmemoración del décimo aniversario de la celebración de la Conferencia Internacional sobre la Población y el Desarrollo: acciones emprendidas para la implementación del programa de acción en América Latina y el Caribe, CEPAL, Serie Población y Desarrollo No. 55, junio.

(2003), La situación de las personas mayores, Santiago de Chile, CEPAL, Conferencia regional intergubernamental sobre envejecimiento, DDR/1.

(2002), Vulnerabilidad sociodemográfica: viejos y nuevos riesgos para comunidades, hogares y personas, Brasilia, CEPAL, Vigesimonoveno período de sesiones, LC/R.2086.

Chackiel (2004), La dinámica demográfica en América Latina, Santiago de Chile, CEPAL, serie Población y Desarrollo, 52, mayo.

(2000), El envejecimiento de la población latinoamericana: ¿hacia una relación de dependencia favorable?, CEPAL, serie Población y Desarrollo, No. 4, mayo.

Chackiel, J. y R. Plaut (1994), "América Latina: tendencias demográficas con énfasis en la mortalidad", Notas de Población, No. 60, pp. 11-46.

Chackiel, J. y S. Schkolnik (2004), "Los sectores rezagados en la transición de la fecundidad en América Latina”, Revista de la CEPAL, No. 83 (agosto), pp. 13-31. Chesnais, J. (1992), The Demographic Transition, Oxford, Clarendon Press.

(1990), El proceso de envejecimiento de la población, Santiago de Chile, Centro Latinoamericano de Demografía, LC/DEM/G.87. 
Coale, A. y S. Watkins (1986) The decline of fertility in Europe, Princeton, Princeton University Press.

Easterlin, R. (1985), The Fertility Revolution; A Supply and Demand Analysis, Chicago, University of Chicago Press.

González, G. y otros (1982), Estrategias de desarrollo y transición demográfica, Santiago, Centro Latinoamericano de Demografía (CELADE).

Guzmán (1997), "El aporte latinoamericano al análisis de los factores determinantes de la fecundidad", Notas de Población, No. 66, pp. 87-109.

Guzmán, J. (1996), "Social change and the fertility decline in Latin America" en J. Guzmán et al., The Fertility Transition in Latin America, Oxford, Clarendon Press.

Guzmán, J. y J. Rodríguez (1993), "La fecundidad pre-transicional en América Latina: un capítulo olvidado", Notas de Población, No. 57.

Guzmán, J. y otros (2001), Diagnóstico de salud sexual y reproductiva de los adolescentes en América Latina y el Caribe, Ciudad de México, Fondo de Población de las Naciones Unidas.

Kirk, D. (1996), “The demographic transition”, Population Studies, vol. 50, No. 3, pp. 361-387.

Rodríguez, J. (2003), La fecundidad alta en América Latina y el Caribe: un riesgo en transición, CEPAL, Serie Población y Desarrollo, No. 55, junio.

(2001), Vulnerabilidad y grupos vulnerables: un marco de referencia conceptual mirando a los jóvenes, CEPAL, Serie Población y Desarrollo, No. 17 , noviembre.

(2000), Vulnerabilidad demográfica: una faceta de las desventajas sociales, CEPAL, Serie Población y Desarrollo, No. 5, junio.

OPS (2002), La salud en las Américas, Washington, Organización Panamericana de la Salud.

Soto, Z. (1978), América Latina: actividades desarrolladas por los programas de planificación familiar, 1976, Santiago, Centro Latinoamericano de Demografía.

Tabah, L. (1989), "From one demographic transition to another", Population Bulletin of the United Nations, No. 28, pp. 1-24.

Timæus, I., J. Chackiel y L. Ruzicka (1996), Adult Mortality in Latin America, Oxford, Clarendon Press.

United Nations (2003), World Population Prospects: The 2002 Revision, New York, DESA/Population Division (ST/ESA/SER.A/22). 
DESA/Population Division (ST/ESA/SER.A/93/Add.1).

Urzúa, R. (1979), El desarrollo y la población en América Latina, Ciudad de México, Siglo XXI Editores.

van de Kaa, D. (2001), "Postmodern fertility preferences: from changing value orientations to new behavior", en R. Bulatao y D. Casterline (eds.), Global Fertility Transition, suplemento especial del volumen $27 \mathrm{de}$ Population and Development Review

(1997), "Narraciones ancladas: historia y resultados de medio siglo de investigaciones sobre los determinantes de la fecundidad", Notas de Población, No. 66, pp. 9-85.

(1994), "The second demographic transition revisited: theories and expectations", en G. Beets et al. (comps.), Population and Family in the Low Countries, Lisse, Sweta and Zeitlinger.

Villa, M. y D. González (2004), La transición demográfica de América Latina y algunos retos en materia de población y desarrollo, México, D. F., Consejo Nacional de Población (CONAPO).

(2003), América Latina y el Caribe: un caso de transición demográfica heterogénea, Asunción, Asociación de Estudios de Población del Paraguay.

Villa, M. y L. Rivadeneira (2000), "El proceso de envejecimiento de la población de América Latina y el Caribe: una expresión de la transición demográfica", Encuentro latinoamericano y caribeño sobre las personas de edad, Santiago de Chile, CEPAL, Serie de Seminarios y Conferencias, No. 2. 